\title{
Pfaffian Bundles and the Ising Model
}

\author{
John Palmer* \\ Department of Mathematics, University of Arizona, Tucson, Arizona 85721, USA
}

\begin{abstract}
An infinite volume Pfaffian formalism is developed for the Ising model.
\end{abstract}

\section{Introduction}

In this paper we will establish a connection between the Pfaffian formalism for the Ising model and the transfer matrix formalism. In [H] Hurst makes the connection between the Pfaffian formalism and the transfer matrix formalism for the Ising model. The formalism he employs is not suited to a direct infinite volume analysis. Working with Grassmann integrals Sato, Miwa, and Jimbo [SMJ] have also worked out such a connection for a class of models they refer to as orthogonal models. The Grassmann integrals they employ only make sense in finite dimensions. Our main interest here is in formulating a direct connection in the thermodynamic limit where the relevant vector spaces are infinite dimensional.

The Pfaffian approach to the Ising model produces a formula for the partition function on a finite lattice as the Pfaffian (or sum of Pfaffians) of a finite dimensional skew symmetric matrix (see McCoy and $\mathrm{Wu}[\mathrm{MW}]$ ). In the infinite volume limit this skew symmetric matrix becomes a finite difference operator on $l^{2}\left(Z_{1 / 2}^{2}, R^{4}\right)$. The finite volume correlation functions are ratios of Pfaffians of operators with similar structure except that the numerator has inhomogeneities that depend on the $n$ sites in the correlation function. It is difficult to rigorously control the infinite volume limit in the Pfaffian approach and we will not attempt to do so here. There is another approach to the Ising correlations where the thermodynamic limit has been rigorously treated [PT]. This is the original transfer matrix formalism of Onsager and Kaufmann $[\mathrm{O}, \mathrm{K}]$. In this paper we will start with the problem of understanding the Pfaffian for a family of skew symmetric operators on a Hilbert space, and we will then make the connection between this problem and the infinite volume transfer matrix formalism. We will have then established a Pfaffian

\footnotetext{
* Research supported in part by NSF grant DMS-8421289
} 
formalism for the Ising model which applies directly in the infinite volume limit.

Section 1 of this paper provides a definition of the Pfaffian bundle over the skew Fredholm maps on the complexification of a real Hilbert space. The idea for this definition comes from the definition of relative Pfaffians in Pressley and Segal's book on loop groups [PS] and Quillen's definition of the determinant bundle over the Fredholm maps between Hilbert spaces [Q]. There has recently been other work on relative Pfaffians [JLW] technically more refined than what appears in Sect. 1.

Section 2 of this paper gives a quick description of the spin bundle over the restricted orthogonal group and the Pfaffian bundle over the isotropic Grassmannian. Although not usually formulated in these terms this is the natural setting for the transfer matrix formalism for the Ising model. In this part we follow mostly [CP], though Pressley and Segal's account of the spin group was also very helpful.

Section 3 introduces the family of finite difference operators that will be the object of attention in the rest of the paper. We make the connection with the transfer matrix formalism by using some ideas in Witten [W]. Apart from the introduction of the transfer matrix the principal result of this section is the proof that the family of operators we consider consists of Fredholm operators of index 0 .

The final section of this paper introduces truncated versions of the finite difference operators and uses them to sketch a different proof of the Fredholm property again following some ideas in Witten [W]. The principal result in this section is a proof that the Pfaffian bundle over a certain family of finite difference operators is naturally isomorphic to the spin bundle over the associated transfer matrices (or equivalently to a sub-bundle of the Pfaffian bundle over an isotropic Grassmannian). Once again there is clearly some connection with ideas in Witten [W], but in this case I am not sure how to make them precise. The infinite volume transfer matrix formalism in [PT] may be reinterpreted as giving formulas for the infinite volume correlation functions for the Ising model in terms of a distinguished trivialization of the spin bundle mentioned above. The fact that this spin bundle is isomorphic to the Pfaffian bundle over a family of finite difference operators means that it makes good mathematical sense to think of Ising correlations as relative Pfaffians of the finite difference operators involved. There are a number of advantages to the Pfaffian formalism that make this connection desirable. Certain symmetries such as rotation by $\pi / 2$ (combined with a suitable change in the Boltzmann weights) are invisible in the transfer matrix formalism but are manifest in the Pfaffian formalism. Ising correlations are associated with "strings" in Pfaffian formalism, and the fact that the correlations depend only on the endpoints of the strings is an expression of $Z / 2 Z$ gauge invariance in the Pfaffian view of things but is not easily described in the transfer matrix picture. Finally the study of the dependence of the theory on Boltzmann parameters (allowed to vary in the complex plane) is simpler in the Pfaffian picture because the Hilbert spaces involved in the transfer matrix picture change incommensurately with changes in the Boltzmann variables. The connection between the formalism in [PT] and the trivialization of the spin bundle is described more fully in some remarks following the proof of the main theorem of this section. 
The results of this paper are largely preliminary. It is our hope that much of the structure in integrable models discovered by Baxter and others will translate into interesting gauge "symmetries" in the Pfaffian formalism described here.

\section{The Pfaffian Bundle over Skew Fredholm Maps}

It is useful to start with a discussion of the problem of making sense of the Pfaffian of a skew symmetric operator on a Hilbert space. Because of the close relation between Pfaffians and determinants this is very much like the problem of understanding infinite determinants, and we begin by reviewing the situation for determinants. It is well known that if $L$ is a trace class perturbation of the identity on a Hilbert space $H$, then $\operatorname{det}(L)$ may be defined as a continuous extension of the finite dimensional determinant [S]. In the problem we consider $L$ is not a compact perturbation of the identity. Instead $L$ is merely a Fredholm operator of index 0 . Such operators are invertible modulo the trace class. One can find an invertible $q$ so that $L q^{-1}$ is a trace class (or even finite rank) perturbation of the identity. One might then define a "relative" determinant for $L$ as $\operatorname{det}\left(L q^{-1}\right)$. For a single operator $L$ this is not very interesting. It produces a number which is 0 when $L$ fails to be invertible but which could be any non-zero complex number when $L$ is invertible depending on the choice of $q$. It becomes a little more interesting when $L$ is a member of a whole family $\mathscr{B}$ of Fredholm operators with index 0 . It may be necessary to choose $q$ so that it depends on the element $L$ in $\mathscr{B}$ which it is to invert modulo the trace class. The formalism of determinant bundles allows one to discuss this problem in a precise manner. Let $\mathscr{F}_{0}$ denote the family of all Fredholm operators on $H$ with index 0 . Living over the base $\mathscr{F}_{0}$ there is a line bundle (the determinant bundle) which captures the ambiguity of regularized determinants in a useful way (see Quillen [Q] and Segal, Wilson [SW]). An element in the fiber over $L$ is a pair $(q, \lambda)$ with $q$ invertible such that $L q^{-1}$ is a trace class perturbation of the identity and $\lambda$ is a complex number. Two such pairs $\left(q_{1}, \lambda_{1}\right)$ and $\left(q_{2}, \lambda_{2}\right)$ are equivalent if and only if $\lambda_{1}=\lambda_{2} \operatorname{det}\left(q_{2} q_{1}^{-1}\right)$. This bundle is constructed so that $L \rightarrow\left(q, \operatorname{det}\left(L q^{-1}\right)\right)$ is well defined and a section as follows directly from the multiplicative property of determinants. This section of the determinant bundle over $\mathscr{F}_{0}$ is called the canonical section $\sigma$. It is clear that $\sigma(L)$ vanishes precisely for those $L$ which fail to be invertible. In order to be able to turn the section $\sigma$ into a function $\operatorname{det}(L)$ with that same property it is clear that one needs a non-vanishing section $\delta$ of the determinant bundle over $\mathscr{B}$. One may then define a determinant as follows:

$$
\sigma(L)=\delta(L) \operatorname{det}_{\delta}(L) .
$$

A non-vanishing continuous section of the bundle $\operatorname{det} \rightarrow \mathscr{B}$ is also called a trivialization. There may be topological obstructions to finding a trivialization (measured by a Chern class). If there is such an obstruction the search for a relative determinant for the family $\mathscr{B}$ is at an end. If the bundle $\operatorname{det} \rightarrow \mathscr{B}$ does have a non-vanishing section, then it will have many such and one requires additional criteria such as gauge invariance, analyticity, or locality to single out a particular choice. In the example we consider the choice of trivialization will be determined 
for "physically" relevant parameters by the requirement that the determinant is the square of the infinite volume correlation functions for the Ising model.

Next we consider the problem of defining Pfaffian bundles. Suppose $H$ is a real Hilbert space with inner product $(\cdot, \cdot)$. The real inner product has a natural complex bilinear extension to the complexification $H_{\mathrm{C}}$ of $H$. We will say that a complex linear map $L$ on $H_{\mathbf{C}}$ is skew symmetric if $(L x, y)=-(x, L y)$. We write $L^{\tau}$ for the transpose of $L$ relative to the bilinear form on $H_{\mathbf{C}}$. To motivate our definitions in the complex case it will be useful to recall some results of Atiyah and Singer for real skew symmetric maps. In [AS] it is shown that the space of real linear skew symmetric Fredholm maps on a real Hilbert space which anti commute with a fixed complex structure has two components distinguished by the parity of the dimension of the null space (this is the space they denote by $\mathscr{F}^{2}$ ). The skew symmetric maps $L$ with even dimensional kernel fall into one path connected component and those with odd dimensional kernel fall into the other component. The space we wish to consider is obtained from the first component of $\mathscr{F}^{2}\left(H_{\mathbf{C}}\right)$ by multiplying by the distinguished conjugation on $H_{\mathbf{C}}$. Let $S k_{0}\left(H_{\mathbf{C}}\right)$ denote the space of skew symmetric Fredholm maps on $H_{C}$ that have an even dimensional null space. We will now define a holomorphic line bundle, $P f$, over the base $S k_{0}\left(H_{\mathrm{C}}\right)$ which we refer to as the Pfaffian bundle. We will imitate Quillen's definition of determinant bundles [Q] in order to exhibit clearly the holomorphic structure of the Pfaffian bundle (it is not evident in our oversimplified account but the determinant bundle is a holomorphic line bundle). Let $F$ denote a finite rank skew symmetric map on $H_{\mathbf{C}}$. Let $U_{F}=\left\{L \in S k_{0}\left(H_{\mathbf{C}}\right): L+F\right.$ is invertible $\}$. It is clear that $U_{F}$ is open in $S k_{0}\left(H_{C}\right)$. We will show that the collection $\left\{U_{F}\right\}$ with $F$ ranging over all finite rank skew symmetric maps on $H_{\mathbf{C}}$ is a covering of $S k_{0}\left(H_{\mathbf{C}}\right)$ and we will give holomorphic transition functions on the intersection of two such sets to define the bundle $P f$.

Lemma. If $L \in S k_{0}\left(H_{\mathbf{C}}\right)$, then there exists a finite rank skew symmetric map $F$ on $H_{\mathbf{C}}$ such that $L+F$ is invertible.

Proof. Let $P$ denote the distinguished conjugation on $H_{\mathrm{C}}$ which fixes $H$. The space $H_{\mathrm{C}}$ is naturally a Hilbert space with respect to the Hermitian inner product $\langle x, y\rangle:=(P x, y)$. Let $L^{*}$ denote the Hermitian conjugate of a linear map $L$ with respect to the inner product $\langle\cdot, \cdot\rangle$. If $L \in S k_{0}\left(H_{\mathrm{C}}\right)$ then $L^{\tau}=-L$ so the Fredholm index of $L$ and that of $L^{\tau}$ are the same. But the Fredholm index of $L^{\tau}$ and $L^{*}$ are the same since they differ by conjugation by $P$. The index of $L^{*}$ is minus the index of $L$ and this implies then that the index of $L$ must be zero. Let $\operatorname{ker}(L)$ denote the null space of $L$ and write coker $(L)$ for the quotient of $H_{\mathrm{C}}$ by $R(L)$, the range of $L$. Each element $x$ in $\operatorname{ker}(L)$ induces a linear functional $(x, \cdot)$ on coker $(L)$ which maps $y+R(L)$ into $(x, y)$. This is well defined since $(x, L y)=-(L x, y)=0$ if $x \in \operatorname{ker}(L)$. The map $x \rightarrow(x, \cdot)$ is injective since $(x, \cdot)=0$ implies that $x$ is complex orthogonal to all of $H_{\mathrm{C}}$ and hence 0 . But since the index of $L$ is 0 the space $\operatorname{ker}(L)$ and the dual of coker $(L)$ have the same dimension and the map $x \rightarrow(x, \cdot)$ is an isomorphism between $\operatorname{ker}(L)$ and the dual space coker $(L)^{*}$. Now choose a complement $K$ to $R(L)$ in $H_{\mathrm{C}}$ on which the bilinear form $(\cdot, \cdot)$ is non-degenerate. For $x \in \operatorname{ker}(L)$ the linear functional $(x, \cdot)$ may be identified with a linear functional on $K$ and the map from $\operatorname{ker}(L)$ to the dual space $K^{*}$ is an isomorphism. Because the bilinear form $(\cdot, \cdot)$ is 
non-degenerate on $K$ it follows that the set of vectors in $H_{\mathrm{C}}$ orthogonal to $K$ relative to the bilinear form, for which we write $K^{\perp}$, is a complement to $K$. The codimension of $K^{\perp}$ is thus $\operatorname{dim}(K)=\operatorname{dim}(\operatorname{ker}(L))$. Since $\operatorname{ker}(L) \cap K^{\perp}=\{0\}$ it follows that $H_{\mathrm{C}}$ is the direct sum $\operatorname{ker}(L)+K^{\perp}$. We now define a finite rank skew symmetric map $F$ on $H_{\mathrm{C}}$ so that $L+F$ is invertible. Let $\left\{e_{1}, e_{2}, \ldots, e_{2 n}\right\}$ be a basis for $\operatorname{ker}(L)$ (which is even dimensional since $\left.L \in S k_{0}\left(H_{\mathrm{C}}\right)\right)$. Let $\left\{e_{1}^{*}, e_{2}^{*}, \ldots, e_{2 n}^{*}\right\}$ denote the dual basis of $K$ (i.e., $\left.\left(e_{j}, e_{k}^{*}\right)=\delta_{j k}\right)$. Let $\left(f_{j k}\right)_{j, k=1,2, \ldots, 2 n}$ denote any invertible skew symmetric $2 n$ by $2 n$ matrix and define:

$$
F e_{j}=\sum_{k=1}^{2 n} f_{k j} e_{k}^{*} \quad \text { for } \quad j=1,2, \ldots, 2 n, \quad F y=0 \text { for } y \in K^{\perp} .
$$

It is a simple matter to check that the linear extension of $F$ to $\operatorname{ker}(L)+K^{\perp}$ is complex skew symmetric on $H_{\mathbf{C}}$. It is clear that $L+F$ does not have a null space and being Fredholm of index 0 it is also invertible. QED

The lemma just proved shows that the open sets $U_{F}$ cover $S k_{0}\left(H_{\mathbf{C}}\right)$. We now introduce the transition functions for the Pfaffian bundle. Over $U_{F}$ the map $U_{F} \ni L \rightarrow L+F$ is a smooth choice of a skew symmetric map $q=L+F$ which inverts $L$ up to a finite rank perturbation of the identity. If we attempt to imitate the description of the determinant bundle and think of the fibers of the Pfaffian bundle as pairs $(q, \lambda) \lambda \in \mathbf{C}$, then it is natural to define a trivialization of $P f$ over $U_{F}$ by $U_{F} \times \mathbf{C} \ni(L, \lambda) \rightarrow(L+F, \lambda) \in P f$. To define the bundle $P f$ we must now say how two such trivializations are related. Suppose $F$ and $G$ are both finite rank complex skew symmetric maps on $H_{\mathrm{C}}$. Proceeding informally we would like the transition function from the $U_{F}$ trivialization to the $U_{G}$ trivialization to incorporate the notion of equivalence $\left(L+F, \lambda_{F}(L)\right) \sim\left(L+G, \lambda_{G}(L)\right)$ if and only if:

$$
\lambda_{G}(L)=\lambda_{F}(L) \frac{P f(L+F)}{P f(L+G)} .
$$

The ratio of Pfaffians $P f(L+F) / P f(L+G)$ makes sense in finite dimensions but it does not directly make sense in infinite dimensions (note: the definition of a Pfaffian for a skew map on a finite dimensional $H_{\mathrm{C}}$ requires a choice of volume form on $H_{\mathrm{C}}$; this ambiguity disappears in the ratios of Pfaffians that we are concerned with here). As a substitute for this undefined ratio of Pfaffians we follow [PS, Chap. 15] to see that $\operatorname{det}\left((L+F)(L+G)^{-1}\right)$ has a canonical square root.

Let us recall some of the results from [PS]. Suppose for the moment that $H_{\mathrm{C}}$ is finite dimensional and that $S$ and $T$ are skew symmetric maps on $H_{\mathbf{C}}$. Let $\left\{e_{1}, e_{2}, \ldots, e_{n}\right\}$ denote a self dual basis for $H_{\mathrm{C}}$ relative to the bilinear form on $H_{\mathrm{C}}$. The matrices of $S$ and $T$ relative to such a self dual basis are skew symmetric. For any finite subset $\sigma$ of the integers from 1 to $n$ let $S_{\sigma}$ denote the skew submatrix of $S$ made from the rows and columns of $S$ indexed by $\sigma$. Define:

$$
P f(I-S T)=\sum_{\sigma} P f\left(S_{\sigma}\right) P f\left(T_{\sigma}\right),
$$

where the sum ranges over all finite subsets $\sigma$ of the integers from 1 to $n$. In [PS] it is shown that:

$$
P f(I-S T)^{2}=\operatorname{det}(I-S T)
$$


Return now to the consideration of infinite dimensional $H_{\mathrm{C}}$. For $S$ and $T$ in the Schmidt class the formula given above can be shown to converge absolutely [PS]. We require a somewhat different infinite dimensional extension to maps $S$ in the trace class and maps $T$ which are merely bounded. Since we do not know whether the formula given above converges in this situation we proceed somewhat differently. Suppose first that $S$ is a finite rank skew symmetric map on $H_{\mathrm{C}}$ and $T$ is a bound skew symmetric map on $H_{\mathrm{C}}$. Let $W$ denote a finite dimensional subspace of $H_{\mathrm{C}}$ which contains the range of $S$ and on which the bilinear form is non-degenerate. For example, since $P^{2}=1$ such a subspace is given by $R(S)+P R(S)$ (recall that $P$ is the distinguished conjugation on $H_{\mathrm{C}}$ ). Because $W$ is non-degenerate the orthogonal complement, $W^{\perp}$, of $W$ with respect to the bilinear form on $H_{\mathrm{C}}$ is a complement to $W$ in $H_{\mathrm{C}}$. Since $S$ is skew we have for $x \in H_{\mathrm{C}}$ and $y \in W^{\perp}: 0=$ $(S x, y)=-(x, S y)$ which implies that $S y=0$ for all $y \in W^{\perp}$, and we conclude that $W^{\perp} \subset \operatorname{ker}(S)$. Let $\pi$ denote the projection on $W$ along $W^{\perp}$. Then $\pi$ satisfies the following two conditions: (1) $\pi$ is a complex orthogonal projection whose range is a non-degenerate subspace which contains the range of $S$; and (2) the range of $I-\pi$ is contained in $\operatorname{ker}(S)$. Suppose $\pi$ is any projection satisfying the conditions (1) and (2) above. Let $S(\pi)=\pi S \pi$ and $T(\pi)=\pi T \pi$ denote the compression of $S$ and $T$ to the subspace $R(\pi)$. Since $\pi$ is a complex orthogonal projection it follows that $\pi^{\tau}=\pi$. Thus $S(\pi)$ and $T(\pi)$ are skew symmetric on $R(\pi)$. Since $R(\pi)$ is non-degenerate it has a self dual basis and we define:

$$
P f(I-S T):=P f(I-S(\pi) T(\pi)) .
$$

On the right the maps $I, S(\pi)$, and $T(\pi)$ are all regarded as transformations on the finite dimensional subspace $R(\pi)$. To see that this definition makes sense we will show that it does not depend on the particular choice for $\pi$. If $\pi_{1}$ and $\pi_{2}$ are two projections which satisfy conditions (1) and (2) above then the complex orthogonal projection, $\pi$, on the sum of their ranges also satisfies these two conditions. Thus to show that the Pfaffian we have defined for $\pi_{1}$ is the same as that for $\pi_{2}$, it is enough to show that they each agree with the Pfaffian defined for $\pi$. If $R(\pi)$ is not equal to $R\left(\pi_{1}\right)$, then $R(\pi)$ is the direct sum of $R\left(\pi_{1}\right)$ and the complex orthogonal complement of $R\left(\pi_{1}\right)$ in $R(\pi)$. Let $\left\{e_{1}, e_{2}, \ldots, e_{n}\right\}$ be a self dual basis of $R(\pi)$ which respects this splitting with $\left\{e_{1}, e_{2}, \ldots, e_{k}\right\}$ a basis for $R\left(\pi_{1}\right)$. The vectors $e_{k+1}, \ldots, e_{n}$ are then null vectors for $S(\pi)$ and $P f\left(S(\pi)_{\sigma}\right)$ vanishes for any $\sigma$ which contains indices between $k+1$ and $n$ since $S(\pi)_{\sigma}$ is then the matrix of a singular transformation. The sum defining $P f(I-S(\pi) T(\pi))$ thus reduces to that for $P f\left(I-S\left(\pi_{1}\right) T\left(\pi_{1}\right)\right)$. The Pfaffian we have defined is thus independent of the choice of projection $\pi$. We would like to know that $P f(I-S T)^{2}=\operatorname{det}(I-S T)$. To see this observe that since $S T=\pi S T=\pi S(I-\pi+\pi) T=\pi S \pi T$ we have: $\operatorname{det}(I-S T)=$ $\operatorname{det}(I-\pi S \pi T)=\operatorname{det}(I-\pi S \pi T \pi)=\operatorname{det}(I-S(\pi) T(\pi))=P f(I-S T)^{2}$. We have constructed a canonical square root, $P f(I-S T)$, for $\operatorname{det}(I-S T)$ when $S$ is finite rank and both $S$ and $T$ are skew symmetric on $H_{\mathrm{C}}$. This is enough to give us the square root of $\operatorname{det}\left((L+F)(L+G)^{-1}\right)$ we desire since $(L+F)(L+G)^{-1}=$ $I-(G-F)(L+G)^{-1}$. The map $S=G-F$ is finite rank and both $S$ and $T=(L+G)^{-1}$ are skew symmetric. For $F$ a finite rank skew symmetric map define a section $\sigma_{F}$ of $P f$ over $U_{F}$ by $U_{F} \ni L \rightarrow \sigma_{F}(L):=(L+F, 1)$. The bundle $P f \rightarrow S k_{0}\left(H_{\mathrm{C}}\right)$ is now 
defined by giving the transition function relating the sections $\sigma_{F}$ and $\sigma_{G}$ :

$$
\sigma_{G}(L)=P f\left((L+F)(L+G)^{-1} \sigma_{F}(L) \text { for } L \in U_{F} \cap U_{G} .\right.
$$

The formula for $\operatorname{Pf}\left((L+F)(L+G)^{-1}\right)$ makes it quite clear that this is a holomorphic function of $L$. To see that one has actually obtained a line bundle one must check the cocycle conditions for the transition functions. Up to a plus or minus sign these conditions follow from the usual multiplicative property of the determinant and the fact that the square of the Pfaffian is the determinant. The sign ambiguity may be resolved using the fact [PS] that $P f(I-\lambda S T)$ is the unique square root of $\operatorname{det}(I-\lambda S T)$ which is holomorphic in $\lambda$ and equal to 1 at $\lambda=0$.

To conclude this section we extend $P f(I-S T)$ so that it is well defined for trace class skew maps $S$ and bounded skew maps $T$ (what we really use is that the product $S T$ is trace class). This will be useful when we discuss trivializations of sub-bundles of the Pfaffian bundle. Suppose $S$ and $T$ are skew symmetric maps on $H_{\mathrm{C}}$ with $S$ in the trace class and $T$ bounded. Suppose $I-S T$ is invertible. Since $S T$ is compact the set of $\lambda \in \mathbf{C}$ for which $I-\lambda S T$ fails to be invertible is a discrete set of points in $\mathbf{C}$. Thus we can find a smooth simple path $\gamma$ in $\mathbf{C}$ which joins 0 to 1 and avoids any of the points at which $I-\lambda S T$ fails to be invertible. Let $S_{n}$ denote a sequence of finite rank skew symmetric maps which tend to $S$ in trace norm. For $n$ large enough $S_{n} T$ will be arbitrarily close to $S T$ in trace norm and it follows that for $n$ sufficiently large $I-\lambda S_{n} T$ will be invertible for all $\lambda$ on the compact path $\gamma$. Along $\gamma$ one may choose a continuous logarithm for $P f\left(I-\lambda S_{n} T\right)$ which vanishes at $\lambda=0$. One finds:

$$
\log P f\left(I-S_{n} T\right)=\frac{1}{2} \int_{\gamma} \frac{d}{d \lambda} \log \operatorname{det}\left(I-\lambda S_{n} T\right) d \lambda=\frac{1}{2} \int_{\gamma} \operatorname{Tr}\left(\left(I-\lambda S_{n} T\right)^{-1} S_{n} T\right) d \lambda .
$$

Since $\left(I-\lambda S_{n} T\right)^{-1} S_{n} T$ tends uniformly to $(I-\lambda S T)^{-1} S T$ in trace norm for $\lambda$ on $\gamma$ it follows that $P f\left(I-S_{n} T\right)$ converges as $n \rightarrow \infty$ and we may define:

$$
P f(I-S T):=\exp \left[\frac{1}{2} \int_{\gamma} \operatorname{Tr}\left((I-\lambda S T)^{-1} S T\right) d \lambda\right] .
$$

The right-hand side of this equality is a square root for $\operatorname{det}(I-S T)$ even if $S T$ is an arbitrary trace class map. What makes the Pfaffian special is that the result does not depend on the curve $\gamma$. The formula for the Pfaffian clearly shows that it depends analytically on $S$ and $T$ as long as $(I-S T)$ is invertible. We clearly want the Pfaffian to be 0 when $(I-S T)$ fails to be invertible. To see that this extension is analytic in $T$ suppose that $S$ is skew and in the trace class and that $T(z)$ is a holomorphic family of bounded skew maps on $H_{\mathrm{C}}$ defined for $z$ in some connected open subset of the plane. The function $z \rightarrow \operatorname{det}(I-S T(z))$ is holomorphic and so either vanishes identically or has isolated zeros. In the first case there is clearly nothing to prove about the analyticity of the Pfaffian which also vanishes identically. In the second case $P f(I-S T(z))$ is analytic except perhaps at the isolated zeros of the determinant. Since the square of the Pfaffian is the determinant the Pfaffian is clearly continuous at these points and hence analytic. Analyticity in $S$ follows for the same reasons. 


\section{The Pfaffian Bundle over the Isotropic Grassmannian}

In this section we introduce the Pfaffian bundle over the isotropic Grassmannian in an infinite dimensional setting. We follow [PS, Chap. 15] and [CP] and the reader is referred to these references for a more detailed account than we will give here. Let $W:=H_{\mathrm{C}}$ denote the complexification of a real Hilbert space $H$ with the distinguished bilinear form $(\cdot, \cdot)$ obtained from the real inner product on $H$. Write $\langle\cdot, \cdot\rangle$ for the natural Hermitian symmetric inner product on $W$ that makes $W$ into a complex Hilbert space (we take the inner product to be conjugate linear in the first slot). If $L$ is a complex linear map on $W$ we write $L^{\tau}$ for the transpose of $L$ relative to the bilinear form and $L^{*}$ for the Hermitian transpose of $L$ relative to the inner product. A subspaces $V \subset W$ is said to be isotropic if the bilinear form vanishes identically on $V$. Suppose $W_{+}$and $W_{-}$are complementary isotropic subspaces of $W$; we will say that $W_{+}+W_{-}$is an isotropic splitting of $W$. Note that we do assume that an isotropic splitting is a continuous splitting; the projection $Q_{+}$of $W$ on $W_{+}$along $W_{-}$is supposed to be continuous. We write $Q_{-}$for the complementary projection $I-Q_{+}$. Given a linear transformation $L$ on $W$ and an isotropic splitting of $W$ we write:

$$
L=\left(\begin{array}{ll}
A(L) & B(L) \\
C(L) & D(L)
\end{array}\right)
$$

for the matrix of $L$ relative to the decomposition $W=W_{+}+W_{-}$(thus $A(L)$ : $W_{+} \rightarrow W_{+}, B(L): W_{-} \rightarrow W_{+}$, etc.). We say that a map on $W$ is complex orthogonal if it is invertible and preserves the bilinear form on $W$. Let $O_{\text {res }}(W)$ denote the group of complex orthogonals on $W$ which have $\mathrm{B}$ and $\mathrm{C}$ matrix elements in the Schmidt class. Let $S O_{\text {res }}(W)$ denote the connected component of the identity in $O_{\text {res }}(W)$. It is known that an element $S \in O_{\text {res }}(W)$ is in $S O_{\text {res }}(W)$ if and only if the dimension of the null space of $D(S)$ (or of $A(S)$ ) is even [CO]. The Pfaffian bundle we are interested in has the orbit $S O_{\text {res }}(W) W_{-}$as its base. We write $G r_{\text {iso }}=S O_{\text {res }}(W) W_{-}$, and we will refer to this orbit space as the isotropic Grassmannian. This setting is natural for the applications in Sects. 3 and 4, but it is apparently more general than the situation considered in [CP] where the subspaces $W_{+}$and $W_{-}$are assumed to be orthogonal with respect to the Hermitian inner product. It is however, always possible to introduce a metrically equivalent inner product on $W$ so that $W_{+}$and $W_{-}$are Hermitian orthogonal. To see this we first prove that the map $W_{-} \in x \rightarrow(x, \cdot) \in W_{+}^{*}$ (the complex dual of $W_{+}$) is a continuous bijection of Hilbert spaces. A vector $x \in W_{-}$in the kernel of this map is complex orthogonal to $W_{+}$. Since $W_{-}$is isotropic the vector $x$ is complex orthogonal to all of $W$ and hence must be 0 . The map in question is thus injective. To see that it is surjective suppose that $v \in W_{+}^{*}$. Then by the Riesz representation theorem there exists $u \in W$ such that $v(y)=\langle u, y\rangle=(\bar{u}, y)$ where $u \rightarrow \bar{u}$ is the natural conjugation on $H_{\mathbf{c}}$. Now write $\bar{u}=v_{+}+v_{-}$, where $v_{+} \in W_{+}$and $v_{-} \in W_{-}$so that $v(y)=\left(v_{+}+v_{-}, y\right)=\left(v_{-}, y\right)$ since $W_{+}$is isotropic. This finishes the proof that $x \rightarrow(x, \cdot)$ induces an isomorphism between $W_{-}$and $W_{+}^{*}$. We choose an orthonormal basis $e_{k}$ for $W_{+}$and let $e_{k}^{*}$ denote the basis of $W_{-}$that maps into the basis dual 
to $e_{k}$ under the isomorphism just described. Define a new conjugation $P$ on $W$ by:

$$
P \sum_{k}\left(a_{k} e_{k}+b_{k} e_{k}^{*}\right)=\sum_{k}\left(\bar{a}_{k} e_{k}^{*}+\bar{b}_{k} e_{k}\right)
$$

One may check that $W \ni x, y \rightarrow(P x, y)$ is a positive definite Hermitian inner product on $W$ metrically equivalent to $\langle\cdot, \cdot\rangle$ with the further property that $W_{+}$and $W_{-}$ are now orthogonal subspaces. In the rest of this section we suppose that the inner product on $W$ has been adjusted so that $W_{+}$and $W_{-}$are orthogonal. There is a succinct characterization of $G r_{\text {iso }}$ in this case. An isotropic subspace $U$ belongs to $G r_{\text {iso }}$ if and only if the Hermitian orthogonal projections on $U$ and $W_{-}$differ by a Schmidt class operator and the intersection $U \cap W_{+}$is even dimensional.

In order to give a "geometric" realization of the Pfaffian bundle we first introduce the infinite spin group $\operatorname{S} \operatorname{pin}(W)[\mathrm{CP}]$. This group is an extension of $S O_{\text {res }}(W)$ by $\mathbf{C}^{*}$. There is a surjective homomorphism $T: \hat{\operatorname{Sp}} \operatorname{pin}(W) \rightarrow S O_{\text {res }}(W)$ with kernel $\mathbf{C}^{*}$. In finite dimensions the elements of the spin group are naturally associated to automorphisms of the Clifford algebra over $W$ which extend orthogonal transformation on $W$. The homomorphism $T$ assigns to each element in the spin group the corresponding orthogonal transformation on $W$. The situation in the infinite dimensional case is similar but depends more strongly on a distinguished representation of $\operatorname{Spin}(W)$ that we now describe. If $\mathscr{D}$ is a linear space let $L(\mathscr{D})$ denote the space of linear maps from $\mathscr{D}$ into $\mathscr{D}$. The group $\hat{\operatorname{Spin}}(W)$ has a representation in $\Lambda\left(W_{+}\right)$, the complex alternating tensor algebra over $W_{+}$, in the following sense. There exists a dense linear domain $\mathscr{D} \subset \Lambda\left(W_{+}\right)$which contains the vacuum vector $1 \in \mathbf{C} \subset \Lambda\left(W_{+}\right)$and a strongly continuous representation $\hat{\Gamma}: \hat{\operatorname{Sp}} \operatorname{pin}(W) \rightarrow L(\mathscr{D})$. The elements $\hat{\Gamma}(g)$ in this representation for which the vacuum vector is an eigenvector are precisely those whose induced rotation $T(g)$ leaves the subspace $W_{-}$invariant. Thus the projective orbit of 1 in the spin representation $\hat{\Gamma}$ can be identified with isotropic Grassmannian. The Pfaffian bundle $P f \rightarrow G r_{\text {iso }}$ is the line bundle over $G r_{\text {iso }}$ whose fiber at $T(g) W_{-}$is the line through $\hat{\Gamma}(g) 1$ in $\Lambda\left(W_{+}\right)$(by analogy with the determinant bundle over the Grassmannian this might more properly be called the Pfaff* bundle - we will ignore this point in what follows). We want to be more explicit about this. Our description makes it clear that the Pfaffian bundle pulls back to the spin bundle $\hat{\operatorname{Spin}}(W) \rightarrow S O_{\text {res }}(W)$ under the map $S O_{\text {res }}(W) \ni G \rightarrow G W_{-}$. Since the maps into $G r_{\text {iso }}$ which we will be concerned with later all factor though $S O_{\text {res }}(W)$ it will suffice for our purposes to give a more explicit description of the spin bundle. Suppose that $G \in S O_{\text {res }}(W)$ and $D(G)$ is invertible. Then for any $g \in \hat{\operatorname{Sp}} \operatorname{pin}(W)$ with $T(g)=G$ we have $[\mathrm{CP}]$ :

$$
\langle g\rangle:=\langle 1, \hat{\Gamma}(g) 1\rangle \neq 0 \text {. }
$$

Thus for such a $G$ there is a unique element $\sigma_{0}(G) \in \widehat{\operatorname{S}} \operatorname{pin}(W)$ which maps into $G$ under $T$ and which is normalized so that $\left\langle\sigma_{0}(G)\right\rangle=1$. We now generalize this to give trivializations of the spin bundle for an open covering of $S O_{\text {res }}(W)$. Suppose $c: W_{+} \rightarrow W_{-}$is finite rank and skew symmetric $\left(c=-c^{\tau}\right)$. Then the map $\gamma_{c}$ with matrix $\left(\begin{array}{ll}1 & 0 \\ c & 1\end{array}\right)$ is complex orthogonal. In [CP, Lemma 1.3] it is shown that for 
any $G \in S O_{\text {res }}(W)$ it is possible to find such a complex orthogonal $\gamma_{c}$ with $c$ finite rank so that the $D$ matrix element $D\left(\gamma_{c} G\right)=D(G)+c B(G)$ is invertible. It is not hard to see that $\hat{\gamma}_{c}:=\sigma_{0}\left(\gamma_{c}\right)$ leaves the vacuum invariant and indeed that $\gamma_{c} \rightarrow \hat{\gamma}_{c}$ is a homomorphism. For a fixed finite rank skew symmetric map $c: W_{+} \rightarrow W_{-}$let $U_{c}$ denote the open subset of $S O_{\text {res }}(W)$ which contains those elements $G$ with $D(G)+c B(G)$ invertible. Over $U_{c}$ we define a trivialization $U_{c} \ni G \rightarrow \sigma_{c}(G)$ by normalizing $\sigma_{c}(G)$ so that $\left\langle\hat{\gamma}_{c} \sigma_{c}(G)\right\rangle=1$. Suppose $b$ and $c$ are both finite rank skew symmetric maps from $W_{+}$to $W_{-}$. We wish to compare $\sigma_{b}$ with $\sigma_{c}$ on $U_{b} \cap U_{c}$. If $\sigma_{b}(G)=r \sigma_{c}(G)$, then clearly $r=\left\langle\hat{\gamma}_{c} \sigma_{b}(G)\right\rangle=\left\langle\hat{\gamma}_{c} \hat{\gamma}_{b}^{-1} \hat{\gamma}_{b} \sigma_{b}(G)\right\rangle=\left\langle\hat{\gamma}_{c}-b \hat{\gamma}_{b} \sigma_{b}(G)\right\rangle$. Using formula 3.2 in [CP] together with $\left\langle\hat{\gamma}_{b-c}\right\rangle=1$ and $\left\langle\hat{\gamma}_{b} \sigma_{b}(G)\right\rangle=1$ one finds:

$$
r=\frac{\sigma_{b}(G)}{\sigma_{c}(G)}=P f\left(I-(b-c) B(D+b B)^{-1}\right)=P f\left((D+c B)(D+b B)^{-1}\right),
$$

where $B=B(G)$ and $D=D(G)$. The map $B(D+b B)^{-1}$ is skew symmetric because $\gamma_{b} G$ is complex orthogonal. The Pfaffian in this last formula is understood in much the same fashion as the Pfaffians in the first section [PS, CP]. The transition function we have obtained here will suffice for our applications.

\section{A Family of Finite Difference Operators}

In this section we will introduce a family of finite difference operators acting on $H_{\mathrm{C}}:=l^{2}\left(\mathbf{Z}_{1 / 2}^{2}, \mathbf{C}^{4}\right)$ in terms of which one can formulate Ising model correlations (and generalizations). We adapt some ideas in Witten [W] by introducing truncated versions of these finite difference operators obtained by imposing boundary conditions. The truncated operators are a useful tool in our analysis and they also provide a natural setting for the introduction of the transfer matrix formalism.

Let $H:=l^{2}\left(\mathbf{Z}_{1 / 2}^{2}, \mathbf{R}^{4}\right)$ denote the real Hilbert space of $\mathbf{R}^{4}$ valued functions on $\mathbf{Z}_{1 / 2}^{2}$ with the inner product:

$$
(F, G)=\sum_{s \in \mathbf{Z}_{1 / 2}^{2}} F(s) \cdot G(s)
$$

where $F(s) \cdot G(s)$ is the usual inner product in $\mathbf{R}^{4}$. Let $H_{\mathbf{C}}$ denote the complexification of $H$ which we identify with $l^{2}\left(\mathbf{Z}_{1 / 2}^{2}, \mathbf{C}^{4}\right)$. The space $H_{\mathbf{C}}$ is naturally a Hilbert space with Hermitian inner product $\langle F, G\rangle:=\sum_{s} \bar{F}(s) \cdot G(s)$ and distinguished bilinear form $(F, G):=\sum_{s} F(s) \cdot G(s)$. Suppose $F \in H_{\mathrm{C}}$ and define horizontal and vertical translation operators by $t_{1} F(k, l)=F(k-1, l)$ and $t_{2} F(k, l)=F(k, l-1)$. The skew symmetric (with respect to $(\cdot, \cdot)$ ) finite difference operators $L$ on $H_{\mathrm{C}}$ that we are interested in all have the form:

where

$$
\left(\begin{array}{cc}
a_{1} & b \\
-b^{\tau} & a_{2}
\end{array}\right)
$$

$$
a_{j}=\left(\begin{array}{cc}
0 & u_{j}+v_{j} t_{j} \\
-u_{j}-t_{j}^{-1} v_{j} & 0
\end{array}\right)
$$


for $j=1,2$, and

$$
b=\left(\begin{array}{ll}
b_{11} & b_{12} \\
b_{21} & b_{22}
\end{array}\right) .
$$

We suppose that the coefficients $u_{j}, v_{j}$, and $b_{i j}$ are all bounded functions on $Z_{1 / 2}^{2}$ regarded as multiplication operators. Rather interesting families of such operators arise in what Baxter refers to as Z-invariant Ising models [B, PA]. We will not attempt to analyse such families here; for simplicity we will immediately confine our attention to families in which $u_{j}$ and $b_{i j}$ are constants and only the coefficients $v_{j}$ vary from site to site on the lattice. To describe the functions $v_{j}$ it will be very useful to regard them as functions on "bonds" rather than "sites" in a manner that we now indicate. Suppose $V_{1}$ is a function which assigns a complex number to each horizontal bond in $Z_{1 / 2}^{2}$. Let $\beta_{1}(k, l)$ denote the horizontal bond which joins $(k-1, l)$ to $(k, l)$ and write:

$$
v_{1}(k, l)=V_{1}\left(\beta_{1}(k, l)\right) .
$$

In a similar fashion let $V_{2}$ denote a complex function on vertical bonds and write:

$$
v_{2}(k, l)=V_{2}\left(\beta_{2}(k, l)\right)
$$

where $\beta_{2}(k, l)$ is the vertical bond which joins $(k, l-1)$ to $(k, l)$. We will always think of the "site" functions $v_{j}$ as arising from "bond" functions $V_{j}$ in precisely this fashion.

The special choices $u_{j}=1$ for $j=1,2, b=\left(\begin{array}{cc}1 & -1 \\ 1 & 1\end{array}\right)$ and $v_{j}=\tanh \left(K_{j}\right)$ for $j=1,2$ are the Ising parameters for the model with horizontal bond strength $K_{1}$ and vertical bond strength $K_{2}$ (see [MW]). Based on what happens on a finite lattice one would like to identify the free energy per site for this model as the Pfaffian $P f(L)$. To make sense of this infinite Pfaffian one can pull back the Pfaffian bundle over $S k_{0}\left(H_{\mathrm{C}}\right)$ by the map $\left(v_{1}, v_{2}\right) \rightarrow L$. A suitable trivialization of the resulting bundle over the "space of Boltzmann weights" then gives the free energy per site of this model. This view of the free energy result admittedly makes something simple seem complicated. For more complicated models such as Baxter's Z-invariant generalization of the Ising model, the "Pfaffian bundle over the space of Boltzmann weights" might be the appropriate object in which to frame a direct infinite dimensional analysis. As previously mentioned we will not pursue this here.

Instead we concentrate on the Pfaffians that appear in the study of spin correlation functions. To incorporate a spin variable at site $(m, n)$ on the integer lattice $Z^{2}$ one introduces a path $\Gamma$ on the integer lattice which joins $(m, n)$ to " $\infty$." A path in $Z^{2}$ we take to be a sequence of directed bonds such that the "head" of each bond matches the "tail" of the succeeding bond. We say that a path, $\Gamma$, joins $(m, n)$ to $\infty$ if the bonds in the sequence eventually leave any bounded subset of the plane. A path $\Gamma$ will be said to be regular if any bond in the sequence $\Gamma$ appears only finitely many times. Suppose now that $\Gamma$ is a regular path in $Z^{2}$ which joins $(m, n) \in Z^{2}$ to $\infty$. Let $v_{j}=t h\left(K_{j}\right)$ as above and define functions $V_{j}^{\Gamma}$ on the bonds in $Z_{1 / 2}^{2}$ by

$$
V_{j}^{\Gamma}(\beta)=(-1)^{N} \Gamma^{(\beta)} v_{j}
$$


where $N_{\Gamma}(\beta)$ is the number of times a bond in the sequence $\Gamma$ crosses the half integer lattice bond $\beta$. Again based on what happens in finite dimensions the expected value of the spin ought to be the relative Pfaffian $\operatorname{Pf}\left(L_{0}^{-1} L_{I}\right)$, where $L_{0}$ is the translation invariant finite difference operator with constant coefficients $V_{j}(\beta):=v_{j}$ and $L_{\Gamma}$ is obtained from $L_{0}$ by replacing $V_{j}$ with $V_{j}^{\Gamma}$. The $p$ site correlations are similar. The single path becomes a union of $p$ different paths, $\Gamma$, each of which joins one of the sites in the correlation to $\infty$. With this alteration in the meaning of $\Gamma$ the definition of $V_{j}^{\Gamma}$ is otherwise unchanged and one would like to identify the $p$ site correlation as the relative Pfaffian $P f\left(L_{0}^{-1} L_{\Gamma}\right)$. Unfortunately $L_{\Gamma}$ is not in general a trace class or even a compact perturbation of $L_{0}$, and so the relative Pfaffians do not make sense directly. Most of the rest of this paper will be devoted to understanding an appropriate sense to give to these relative Pfaffians.

The Ising model parameters can be generalized in what we do without introducing essential complications. We begin by regarding $u_{j}, v_{j} \neq 0$ and $b_{i j}$ as arbitrary complex parameters and we will introduce restrictions on them as they are needed. Suppose that $\left(m_{j}, n_{j}\right) \in Z^{2}(j=1, \ldots, p)$ are sites on the integer lattice labeled so that $n_{1} \leqq n_{2} \leqq \cdots \leqq n_{p}$. Let $\Gamma_{j}$ denote a regular path on $Z^{2}$ which joins $\left(m_{j}, n_{j}\right)$ to $\infty$ and write $\Gamma=\left\{\Gamma_{1}, \Gamma_{2}, \ldots, \Gamma_{p}\right\}$. We are interested in the family of finite difference operators $L_{\Gamma}$ which have constant parameters $u_{j}, b_{i j}$ and $v_{j}(k, l)=V_{j}^{\Gamma}\left(\beta_{j}(k, l)\right.$ ). We will analyse the family $L_{T}$ by making a connection with the horizontal transfer matrix formalism. For this purpose it is convenient to normalize the choice of the paths $\Gamma_{j}$ to be the horizontal paths which emerge to the right of the sites $\left(m_{j}, n_{j}\right)$. That is:

$$
\Gamma_{j}:=\left\{\left(m, n_{j}\right) \in Z^{2}: m \geqq m_{j}\right\} .
$$

We have thus supressed the freedom one has in choosing the paths $\Gamma_{j}$ which is one of the most interesting features of the Pfaffian formalism; we hope to return to this matter in another place. For the present we fix the choice of paths $\Gamma_{j}$ given above.

Following Witten [W] we introduce truncated versions of $L_{\Gamma}$ that remain skew symmetric (this is also clearly related to the idea behind Weyl's analysis of differential operators on infinite intervals which proceeds by analysing approximations on finite intervals obtained by introducing suitable boundary conditions). Let $N$ denote an arbitrary integer and for $F, G \in H_{\mathbf{C}}$ define:

$$
(F, G)_{l>N}=\sum_{k \in Z_{1 / 2}} \sum_{l>N} F(k, l) \cdot G(k, l) .
$$

To unburden the notation write $L:=L_{\Gamma}$. Then a simple calculation shows that:

$$
\begin{aligned}
& (L F, G)_{l>N}+(F, L G)_{l>N} \\
& \quad=\sum_{k \in Z_{1 / 2}} v_{2}\left(k, N+\frac{1}{2}\right)\left[F_{3}\left(k, N+\frac{1}{2}\right) G_{4}\left(k, N-\frac{1}{2}\right)+F_{4}\left(k, N-\frac{1}{2}\right) G_{3}\left(k, N+\frac{1}{2}\right)\right] .
\end{aligned}
$$

Based on this formula it is natural to introduce boundary values

$$
\partial_{N} F(k):=\left(\begin{array}{c}
F_{3}\left(k, N+\frac{1}{2}\right) \\
F_{4}\left(k, N-\frac{1}{2}\right)
\end{array}\right)
$$


for $F \in H_{\mathbf{C}}$ and the bilinear form

$$
(f, g)_{N}=\sum_{k \in Z_{1 / 2}} f(k) \cdot v(k) g(k)
$$

where $v(k):=\left(\begin{array}{cc}0 & v_{2}\left(k, N+\frac{1}{2}\right) \\ v_{2}\left(k, N+\frac{1}{2}\right) & 0\end{array}\right)$ and $f$ and $g$ are in $l^{2}\left(Z_{1 / 2}, \mathbf{C}^{2}\right)$. Recall that we suppose $v_{j} \neq 0$ so this bilinear form is non-degenerate.

We may then write the equation which determines the deviation from skew symmetry in a restriction of $L$ as follows:

$$
(L F, G)_{l>N}+(F, L G)_{l>N}=\left(\partial_{N} F, \partial_{N} G\right)_{N} .
$$

Thus to obtain skew symmetric restrictions of $L$ requires boundary conditions on the domain of $L$ that cause the right-hand side of this last equation to vanish. Let $W_{N}$ denote the vector space $l_{2}\left(Z_{1 / 2}, \mathbf{C}^{2}\right)$ with Hermitian inner product $\langle\cdot, \cdot\rangle_{N}$ defined by:

$$
\langle f, g\rangle_{N}=\sum_{k \in Z_{1 / 2}}\left|v_{2}\left(k, N+\frac{1}{2}\right)\right| \vec{f}(k) \cdot g(k)
$$

The distinguished bilinear form $(\cdot, \cdot)_{N}$ on $W_{N}$ is given by:

$$
(f, g)_{N}=\left\langle P_{N} f, g\right\rangle_{N},
$$

where

$$
P_{N} f(k):=\frac{v_{2}\left(k, N+\frac{1}{2}\right)}{\left|v_{2}\left(k, N+\frac{1}{2}\right)\right|}\left(\begin{array}{ll}
0 & 1 \\
1 & 0
\end{array}\right) \bar{f}(k)
$$

The map $P_{N}$ is conjugate linear and has square equal to $I$. Thus the distinguished bilinear form on $W_{N}$ is obtained from the Hermitian inner product by a conjugation. When $N \neq n_{j}(j=1, \ldots, p)$ we lighten the notation by observing that all the vector spaces $W_{N}$ are isomorphic to $W:=l^{2}\left(Z_{1 / 2}, \mathbf{C}^{2}\right)$ with the Hermitian inner product $\langle f, g\rangle:=\left|v_{2}\right| \sum_{k} \bar{f}(k) \cdot g(k)$ and the bilinear form $(f, g):=\langle P f, g\rangle$ with $P f(k):=$ $\frac{v_{2}}{\left|v_{2}\right|}\left(\begin{array}{ll}0 & 1 \\ 1 & 0\end{array}\right) \bar{f}(k)$.

With appropriate restrictions on the coefficients $u_{j}, v_{j}$ and $b_{i j}$ the relation $L_{\Gamma} F=G$ implies and is implied by a linear inhomogeneous relation between $\partial_{n} F$ and $\partial_{n-1} F$ for all $n \in Z$. This relation defines the transfer matrix and is at the heart of our analysis. To obtain this relation we begin by writing the functions $F, G \in l^{2}\left(Z_{1 / 2}^{2}, \mathbf{C}^{4}\right)$ in two component form $F=\left(\begin{array}{l}f_{1} \\ f_{2}\end{array}\right)$, and $G=\left(\begin{array}{l}g_{1} \\ g_{2}\end{array}\right)$ where $f_{j}, g_{j} \in l^{2}\left(Z_{1 / 2}^{2}, \mathbf{C}^{2}\right)$. The equation $L_{\Gamma} F=G$ becomes in component form:

$$
a_{1} f_{1}+b f_{2}=g_{1}, \quad-b^{\tau} f_{1}+a_{2} f_{2}=g_{2} \text {. }
$$

From which one finds $a_{2} f_{2}=b^{\tau} f_{1}+g_{2}$ and $f_{1}=a_{1}^{-1}\left(g_{1}-b f_{2}\right)$. Combining these two equations:

$$
a_{2} f_{2}=b^{\tau} a_{1}^{-1}\left(g_{1}-b f_{2}\right)+g_{2}=-b^{\tau} a_{1}^{-1} b f_{2}+b^{\tau} a_{1}^{-1} g_{1}+g_{2} .
$$


Now define:

and

$$
S:=u_{2}\left(\begin{array}{cc}
0 & -1 \\
1 & 0
\end{array}\right)-b^{\tau} a_{1}^{-1} b
$$

$$
h:=b^{\tau} a_{1}^{-1} g_{1}+g_{2} .
$$

Then the last equation may be rewritten:

$$
\left(\begin{array}{cc}
0 & v_{2} t_{2} \\
-\hat{v}_{2} t_{2}^{-1} & 0
\end{array}\right) f_{2}=S f_{2}+h
$$

where $\hat{v}_{2}(k, l):=v_{2}(k, l+1)$. Let $V:=\left(\begin{array}{cc}0 & -\hat{v}_{2}^{-1} \\ v_{2}^{-1} & 0\end{array}\right)$ and multiply both sides of the last displayed equation by $V$. One finds:

$$
\left(\begin{array}{c}
F_{3}(\cdot, l+1) \\
F_{4}(\cdot, l-1)
\end{array}\right)=\operatorname{VS}\left(\begin{array}{c}
F_{3}(\cdot, l) \\
F_{4}(\cdot, l)
\end{array}\right)+V h(\cdot, l) .
$$

Now write $\sigma_{+}=\left(\begin{array}{ll}1 & 0 \\ 0 & 0\end{array}\right)$ and $\sigma_{-}=\left(\begin{array}{ll}0 & 0 \\ 0 & 1\end{array}\right)$. Then:

and

$$
\left(\sigma_{+}+\sigma_{-} V S\right)\left(\begin{array}{c}
F_{3}(\cdot, l) \\
F_{4}(\cdot, l)
\end{array}\right)=\partial_{l-\frac{1}{2}} F-\sigma_{-} V h(\cdot, l)
$$

$$
\left(\sigma_{-}+\sigma_{+} V S\right)\left(\begin{array}{c}
F_{3}(\cdot, l) \\
F_{4}(\cdot, l)
\end{array}\right)=\partial_{l+\frac{1}{2}} F-\sigma_{+} V h(\cdot, l) .
$$

Solving for $\partial_{l-\frac{1}{2}} F$ in terms of $\partial_{l+\frac{1}{2}} F$ one finds:

where

$$
\partial_{l-\frac{1}{2}} F=T_{l} \partial_{l+\frac{1}{2}} F+\left(\sigma_{-}-T_{l} \sigma_{+}\right) V h(\cdot, l),
$$

and

$$
T_{l}:=\left(\sigma_{+}+\sigma_{-} V S\right)\left(\sigma_{-}+\sigma_{+} V S\right)^{-1}
$$

$$
V=\left(\begin{array}{cc}
0 & -v_{2}(\cdot, l+1)^{-1} \\
v_{2}(\cdot, l)^{-1} &
\end{array}\right), \quad h=b^{\tau} a_{1}^{-1} g_{1}+g_{2} .
$$

The equation (T) will be the principal tool in our analysis. We will call $T_{l}$ the transfer matrix at level $l$. This usage is not consistent with the terminology in statistical mechanics where the lift of $T_{l}$ into a spin group is called the transfer matrix. However, this abuse of terminology will allow us to avoid always referring to $T_{l}$ as the "induced rotation of the transfer matrix" before we've introduced the element in the spin group which has this induced rotation.

To allow the reader to compare what is happening at this point with more familiar treatments of the Ising model we record the result for the transfer matrix in the translation invariant case of the Ising model (no spin inhomogeneities):

$$
T_{\text {Ising }}=\left[\begin{array}{cc}
\frac{v_{2}\left(1+v_{1} t_{1}\right)\left(1+v_{1} t_{1}^{-1}\right)}{1-v_{1}^{2}} & \frac{-v_{1}\left(t_{1}-t_{1}^{-1}\right)}{1-v_{1}^{2}} \\
\frac{v_{1}\left(t_{1}-t_{1}^{-1}\right)}{1-v_{1}^{2}} & \frac{\left(1+v_{1} t_{1}\right)\left(1+v_{1} t_{1}^{-1}\right)}{v_{2}\left(1-v_{1}^{2}\right)}
\end{array}\right]
$$


In this last result make the substitution $v_{j}=\tanh \left(K_{j}\right)$ and transform the result by the similarity $X(\cdot) X^{-1}$ with $X=\left(\begin{array}{cc}-1 & 1 \\ i & i\end{array}\right)$. Thinking of the translation $t_{1}$ acting in a Fourier series representation replace $t_{1}$ by $e^{i \theta}$. One finds:

$$
T_{\text {Ising }}=\left(\begin{array}{cc}
c_{1} c_{2}^{*}-s_{1} s_{2}^{*} \cos (\theta) & s_{1} \sin (\theta)-i\left(c_{1} s_{2}^{*}-s_{1} c_{2}^{*} \cos (\theta)\right) \\
s_{1} \sin (\theta)+i\left(c_{1} s_{2}^{*}-s_{1} c_{2}^{*} \cos (\theta)\right) & c_{1} c_{2}^{*}-s_{1} s_{2}^{*} \cos (\theta)
\end{array}\right),
$$

where $c_{j}:=\operatorname{ch}\left(2 K_{j}\right), s_{j}:=\operatorname{sh}\left(2 K_{j}\right), c_{j}^{*}:=c_{j} / s_{j}$, and $s_{j}^{*}:=1 / s_{j}$. This should look familiar to Ising model devotees.

We will next discuss some restrictions on the parameters $u_{j}, v_{j}$ and $b$ that have important consequences for the transfer matrix. Let $L_{0}=\left(\begin{array}{cc}a_{1} & b \\ -b^{\tau} & a_{2}\end{array}\right)$ with the constant coefficients $u_{j}, v_{j}$ and $b$. Consider the Fourier transform:

$$
\hat{F}(z, w)=\frac{1}{2 \pi} \sum_{k, l \in Z_{1 / 2}} z^{k} w^{l} F(k, l),
$$

where $z=e^{i \theta_{1}}, w=e^{i \theta_{2}}$ and $\theta_{j}$ is restricted to the interval $-\pi<\theta_{j} \leqq \pi$ in order to give a definite sense to fractional powers of $z$ and $w$. The map $L_{0}$ becomes a multiplication operator after Fourier transform since $t_{1}$ becomes multiplication by $z$ and $t_{2}$ becomes multiplication by $w$. Let $L_{0}(z, w)$ denote the matrix obtained from $L_{0}$ by replacing $t_{1}^{ \pm 1}$ with $z^{ \pm 1}$ and $t_{2}^{ \pm 1}$ with $w^{ \pm 1}$. The criterion for $L_{0}$ to be invertible on $l^{2}$ is then:

$$
\operatorname{det} L_{0}(z, w) \neq 0 \quad \text { for } \quad(z, w) \in S^{1} \times S^{1} .
$$

This is our first restriction on the parameters $u_{j}, v_{j}$ and $b$. Let $c_{j}(u):=\left(u_{j}+v_{j} u\right)$ and $b:=\left(\begin{array}{ll}\alpha & \beta \\ \gamma & \delta\end{array}\right)$, then one finds:

$$
\begin{aligned}
\operatorname{det} L_{0}(z, w)= & c_{1}(z) c_{1}\left(z^{-1}\right) c_{2}(w) c_{2}\left(w^{-1}\right)+\beta \gamma\left(c_{1}(z) c_{2}(w)+c_{1}\left(z^{-1}\right) c_{2}\left(w^{-1}\right)\right) \\
& -\alpha \delta\left(c_{1}(z) c_{2}\left(w^{-1}\right)+c_{1}\left(z^{-1}\right) c_{2}(w)\right)+(\operatorname{det} b)^{2} .
\end{aligned}
$$

The one observation we have to make concerning this formula is that it is clearly invariant under the substitutions $z \leftarrow z^{-1}$ and $w \leftarrow w^{-1}$.

It is interesting to consider what effect (A) has on the transfer matrix. Let $T$ denote the transfer matrix for $L_{0}$. Because $L_{0}$ has constant coefficients $T=T_{l}$ is independent of $l$ in an obvious sense. We may identify $W_{N}$ with $W$ as was done above and introduce the Fourier transform:

$$
\hat{f}(z)=\frac{1}{\sqrt{ } 2 \pi} \sum_{l \in Z_{1 / 2}} z^{l} f(l)
$$

for $f \in W$. After Fourier transform the map $T$ becomes a $2 \times 2$ matrix valued multiplication operator $T(z)$ with entries that are rational functions of $z$. The characteristic equation $\operatorname{det}(w I-T(z))=0$ determines the spectral values $w$ for $T(z)$ when $z \in S^{1}$. It is not too surprising that there is a relation between $\operatorname{det}(w I-T(z))$ and $\operatorname{det} L_{0}(z, w)$. One may check that:

$$
w \operatorname{det} L_{0}(z, w)=v_{2}\left[c_{1}(z) c_{1}\left(z^{-1}\right) u_{2}+\beta \gamma c_{1}(z)-\alpha \delta c\left(z^{-1}\right)\right] \operatorname{det}(w I-T(z)) .
$$


Our second condition on the parameters in $L_{0}$ is:

$$
c_{1}(z) c_{1}\left(z^{-1}\right) u_{2}+\beta \gamma c_{1}(z)-\alpha \delta c_{1}\left(z^{-1}\right) \neq 0 \text { for } z \in S^{1} .
$$

Condition (B) implies that the roots $w$ to $\operatorname{det}(w I-T(z))=0$ for $z \in S^{1}$ are the same as the roots of $w$ det $L_{0}(z, w)=0$ for $z \in S^{1}$. For each fixed $z \in S^{1}$ condition (B) implies that the function $w$ det $L_{0}(z, w)$ is a non-trivial quadratic polynomial in $w$ and so has two complex roots. Since $\operatorname{det} L_{0}(z, w)=\operatorname{det} L_{0}\left(z, w^{-1}\right)$ and $\operatorname{det} L_{0}(z, w) \neq 0$ for $(z, w) \in S^{1} \times S^{1}$, it follows that one root lies strictly inside the unit circle and the other lies strictly outside the unit circle. Conditions (A) and (B) together imply that the transfer matrix $T$ is a bounded linear map and that no point on the unit circle is in the spectrum of $T$.

We return now to the consideration of the inhomogeneous operators $L_{\Gamma}$. It is clear that when $l<n_{1}-1$ or $l>n_{p}+1$ the transfer matrix $T_{l}$ for $L_{\Gamma}$ may be identified with the transfer matrix for $L_{0}$. We will now calculate the change in the transfer matrix which is produced by one of the lines in $\Gamma$. The two transfer matrices that are effected by the line of "discontinuities" $\Gamma_{j}=\left\{\left(m, n_{j}\right): m \geqq m_{j}\right\}$ are $T_{n_{j}+\frac{1}{2}}$ and $T_{n_{j}-\frac{1}{2}}$. In the formula for $T_{l}$ the change occurs only in the factor $V$. Let $\varepsilon_{m}(k):=\operatorname{sgn}(m-k)$ for $m \in Z$ and $k \in Z_{1 / 2}$. Then:

and

$$
V\left(k, n_{j}+\frac{1}{2}\right)=\left(\begin{array}{cc}
0 & -v_{2}^{-1} \\
-\varepsilon_{m_{j}}(k) v_{2}^{-1} & 0
\end{array}\right)
$$

$$
V\left(k, n_{j}-\frac{1}{2}\right)=\left(\begin{array}{cc}
0 & -\varepsilon_{m_{j}}(k) v_{2}^{-1} \\
v_{2}^{-1} & 0
\end{array}\right) .
$$

Incorporating these changes in the appropriate $T_{l}$ one easily sees that:

and

$$
T_{n_{j}+\frac{1}{2}}=\left(\begin{array}{cc}
1 & 0 \\
0 & -\varepsilon_{m_{j}}
\end{array}\right) T
$$

$$
T_{n_{j}-\frac{1}{2}}=T\left(\begin{array}{cc}
-\varepsilon_{m_{j}} & 0 \\
0 & 1
\end{array}\right)
$$

Conditions (A) and (B) above are thus adequate for the existence of the transfer matrix for $L_{\Gamma}$ as well as $L_{0}$. It might help the reader to know that in the Ising case conditions (A) and (B) are satisfied for all values of the parameters $K_{j}$ except the critical values. At the critical parameters 1 is in the spectrum of the transfer matrix.

We next turn to an important property of the transfer matrix $T_{l}: W_{l+\frac{1}{2}} \rightarrow W_{l-\frac{1}{2}}$. The map $T_{l}$ is a complex orthogonal map; one has $\left(T_{l} f, T_{l} g\right)_{l-\frac{1}{2}}=(f, g)_{l+\frac{1}{2}}$. Observe that this is equivalent to:

$$
T_{l}^{\tau}\left(\begin{array}{cc}
0 & v_{2}(\cdot, l) \\
v_{2}(\cdot, l) & 0
\end{array}\right) T_{l}=\left(\begin{array}{cc}
0 & v_{2}(\cdot, l+1) \\
v_{2}(\cdot, l+1) & 0
\end{array}\right) .
$$

To check this make use of the fact that $S^{\tau}=-S$ to calculate $T_{l}^{\tau}$. Multiply on the left by $\left(\sigma_{-}-S V^{\tau} \sigma_{+}\right)$and on the right by $\left(\sigma_{-}+\sigma_{+} V S\right)$ to clear the inverse maps 
from the resulting equation. Make some obvious algebraic simplifications and subtract the right-hand side of the equation from both sides to obtain:

$$
\left(\begin{array}{cc}
0 & v_{2}(\cdot, l) \\
-v_{2}(\cdot, l+1) & 0
\end{array}\right) V S-S V^{\tau}\left(\begin{array}{cc}
0 & -v_{2}(\cdot, l+1) \\
v_{2}(\cdot, l) & 0
\end{array}\right)=0 .
$$

Now consult the definition of $V$ to see that the left-hand side is simply $S-S$. We have confirmed that $T_{l}$ is a complex orthogonal. No doubt a more fundamental understanding of $T_{l}$ would make this property manifest.

Since our restrictions (A) and (B) on the coefficients in $L_{0}$ guarantee that $L_{0}$ is invertible it is natural to compare $L_{\Gamma}$ with $L_{0}$. We will now calculate $L_{0}^{-1} L_{\Gamma}$. The calculation is an instructive use of the transfer matrix idea and will also provide a proof that the operators $L_{\Gamma}$ are Fredholm with index 0 (incidently this Fredholm property fails for the Ising model at the critical point). We begin with an observation concerning the transfer matrix $T$. Recall that the spectrum of $T$ does not contain any points on the unit circle $S^{1}$. Let $W_{+}$denote the spectral subspace for $T$ on which $T$ has spectrum strictly inside the unit circle and $W_{-}$the spectral subspace for $T$ on which $T$ has spectrum strictly outside the unit circle. The projections $Q_{ \pm}$ on $W_{ \pm}$are multiplication operators in the Fourier transform variable $z$ given by:

$$
Q_{ \pm}(z)=\frac{1}{2 \pi i} \int_{S^{1}}\left(w I-T(z)^{ \pm 1}\right)^{-1} d w
$$

Our assumptions (A) and (B) imply that $Q_{ \pm}$are bounded, hence that $W=W_{+}+W_{-}$ is a continuous direct sum decomposition of $W$. Each of the subspaces $W_{+}$is isotropic with respect to the bilinear form on $W$. To see this suppose that $x, y \in W_{+}$. Then since $T$ is complex orthogonal:

$$
|(x, y)|=\left|\left(T^{n} x, T^{n} y\right)\right| \leqq\left\|T^{n} x\right\|\left\|T^{n} y\right\| .
$$

But because $x, y \in W_{+}$, we have $T^{n} x \rightarrow 0$ and $T^{n} y \rightarrow 0$ as $n \rightarrow \infty$. Thus we must have $(x, y)=0$ for $x, y \in W_{+}$. If we replace $T$ with $T^{-1}$ the same argument works for $W_{-}$. We are thus in the setting of Sect. 2.

In order to calculate $L_{0}^{-1} L_{\Gamma}$ we begin with the relation:

$$
\left(L_{\Gamma}-L_{0}\right) F(k, l)=2 v_{2} \sum_{j=1}^{p}\left(\begin{array}{c}
0 \\
H_{j}(k, l)
\end{array}\right),
$$

where $0 \in \mathbf{C}^{2}$ and:

$$
H_{j}(k, l):=\left(\begin{array}{c}
-\theta_{j}(k) F_{4}\left(k, n_{j}-\frac{1}{2}\right) \delta\left(l-n_{j}-\frac{1}{2}\right) \\
\theta_{j}(k) F_{3}\left(k, n_{j}+\frac{1}{2}\right) \delta\left(l-n_{j}+\frac{1}{2}\right)
\end{array}\right)
$$

and $\theta_{j}(k):=\theta\left(k-m_{j}\right)$ with $\theta(k)=1$ for $k>0$ and $\theta(k)=0$ for $k<0$. Next we wish to compute $L_{0}^{-1}\left(L_{\Gamma}-L_{0}\right) F$ using the transfer relation (T). Write $L_{0} G=$ $\left(L_{\Gamma}-L_{0}\right) F$. Then for each $n_{j}$ there are two places where the transfer relation (T) for $G$ is inhomogeneous:

$$
\partial_{n_{j}} G=T_{n_{j}+1 / 2} \partial_{n_{j}+1} G-\left(\begin{array}{c}
0 \\
2 \theta_{j} F_{4}\left(\cdot, n_{j}-\frac{1}{2}\right)
\end{array}\right)
$$


and

$$
\partial_{n_{j-1}} G=T_{n_{j}-1 / 2} \partial_{n_{j}} G+T_{n_{j}-1 / 2}\left(\begin{array}{c}
2 \theta_{j} F_{3}\left(\cdot, n_{j}+\frac{1}{2}\right) \\
0
\end{array}\right) .
$$

For clarity we've written $T_{l}$ for the transfer matrix for $L_{0}$ which can of course be identified with $T$. We can combine the last two relations to obtain:

$$
\begin{aligned}
\partial_{n_{j-1}} G & =T\left(n_{j}-1, n_{j}+1\right) \partial_{n_{j}+1} G+T_{n_{j}-1 / 2} \Theta_{j} \partial_{n_{j}} F, \\
\Theta_{j} & :=\left(\begin{array}{cc}
2 \theta_{j} & 0 \\
0 & -2 \theta_{j}
\end{array}\right),
\end{aligned}
$$

where we've introduced, $T(a, b)$, the transfer matrix which takes one from level $b$ to level $a$ defined by:

$$
\begin{aligned}
& T(a, b):=T_{a+1 / 2} T_{a+3 / 2} \cdots T_{b-1 / 2} \text { for } a<b, \\
& T(a, b):=T(b, a)^{-1} \text { for } a>b, \\
& T(a, a):=I
\end{aligned}
$$

for $a, b \in Z$. Suppose now that $n$ and $N$ are integers chosen so that $n<n_{1}$ and $n_{p}<N$. The transfer relation (T) shows that $\partial_{n} G$ is obtained from $\partial_{N} G$ by repeated application of the transfer matrix with the inhomogeneous modifications just described as one passes through level $n_{j}$. One finds:

$$
\partial_{n} G=T(n, N) \partial_{N} G+\sum_{j=1}^{p} T\left(n, n_{j}\right) \Theta_{j} \partial_{n_{j}} F .
$$

We now come to a crucial element in our calculation. The boundary values $\partial_{s} G$ for $s>N$ are all obtained from $\partial_{N} G$ by repeated application of $T^{-1}$. For $G$ to be in $H_{\mathbf{C}}$ it is necessary and sufficient that $\partial_{N} G$ should lie in the subspace on which $T^{-1}$ acts as a contraction. That is we must have $\partial_{N} G \in W_{-}$. For precisely analogous reasons we must have $\partial_{n} G \in W_{+}$. Thus $Q_{-} \partial_{n} G=0$ and it follows that:

$$
\partial_{N} G=-\sum_{j=1}^{p} T\left(N, n_{j}\right) Q_{-} \Theta_{j} \partial_{n_{j}} F
$$

since $Q_{-}$commutes with the transfer matrix $T$ and $Q_{-} \partial_{N} G=\partial_{N} G$. For reasons we will soon make plain we are especially interested in $\partial_{n_{J}} G$ for which one has:

$$
\partial_{n_{j}} G=T\left(n_{j}, N\right) \partial_{N} G+\sum_{k=j+1}^{p} T\left(n_{j}, n_{k}\right) \Theta_{k} \partial_{n_{k}} F-2 \theta_{j} \sigma_{-} \partial_{n_{j}} F
$$

Substituting the result for $\partial_{N} G$ into the expression for $\partial_{n_{j}} G$ one finds:

$$
\begin{aligned}
\partial_{n_{j}} G= & -2\left(Q_{+} \theta_{j} \sigma_{-}+Q_{-} \theta_{j} \sigma_{+}\right) \partial_{n_{j}} F-\sum_{k=1}^{j-1} T\left(n_{j}, n_{k}\right) Q_{-} \Theta_{k} \partial_{n_{k}} F \\
& +\sum_{k=j+1}^{p} T\left(n_{j}, n_{k}\right) Q_{+} \Theta_{k} \partial_{n_{k}} F .
\end{aligned}
$$

This is nearly the result we desire. We obtain a value for $\partial_{n_{J}}\left(L_{0}^{-1} L_{\Gamma} F\right)$ by adding 
$\partial_{n_{j}} F$ to the result just exhibited:

$$
\begin{aligned}
\partial_{n_{j}}\left(L_{0}^{-1} L_{\Gamma} F\right)= & Q_{+}\left(\begin{array}{cc}
1 & 0 \\
0 & -\varepsilon_{j}
\end{array}\right) \partial_{n_{j}} F+Q_{-}\left(\begin{array}{cc}
-\varepsilon_{j} & 0 \\
0 & 1
\end{array}\right) \partial_{n_{j}} F \\
& -\sum_{k=1}^{j-1} T\left(n_{j}, n_{k}\right) Q_{-} \Theta_{k} \partial_{n_{k}} F+\sum_{k=j+1}^{p} T\left(n_{j}, n_{k}\right) Q_{+} \Theta_{k} \partial_{n_{k}} F .
\end{aligned}
$$

Now let $\partial H_{\mathrm{C}}$ denote the subspace $\sum_{j=1}^{p} \partial_{n_{j}} H_{\mathrm{C}}$, where $\partial_{n_{j}} H_{\mathrm{C}}$ denotes the orthogonal complement in $H_{\mathrm{C}}$ of the kernel of the map $\partial_{n_{\mathrm{j}}}$. Let $\partial H_{\mathrm{C}}^{\perp}$ denote the orthogonal complement of $\partial H_{\mathrm{C}}$. Then relative to the splitting $H_{\mathrm{C}}=\partial H_{\mathrm{C}}+\partial H_{\mathrm{C}}^{\perp}$ the matrix of $L_{0}^{-1} L_{\Gamma}$ is lower triangular $\left(\begin{array}{cc}M & 0 \\ * & I\end{array}\right)$.

Recall that a linear map on a Hilbert space is Fredholm of index 0 if and only if some finite rank perturbation of it is invertible. Thus $L_{0}^{-1} L_{\Gamma}$ (and hence also $L_{\Gamma}$ ) will be Fredholm with index 0 if and only if $M$ is Fredholm with index 0 . The calculation of $\partial_{n_{j}}\left(L_{0}^{-1} L_{\Gamma} F\right)$ given above gives us the matrix representation of $M$ on $\partial H_{\mathrm{C}}=\sum_{j=1}^{p} \partial_{n_{j}} H_{\mathrm{C}}$ :

$$
\begin{aligned}
& M_{j j}=Q_{+}\left(\begin{array}{cc}
1 & 0 \\
0 & -\varepsilon_{j}
\end{array}\right)+Q_{-}\left(\begin{array}{cc}
-\varepsilon_{j} & 0 \\
0 & 1
\end{array}\right), \\
& M_{j k}=T\left(n_{j}, n_{k}\right) Q_{+} \Theta_{k} \text { for } j<k \\
& M_{j k}=-T\left(n_{j}, n_{k}\right) Q_{-} \Theta_{k} \text { for } j>k
\end{aligned}
$$

We will now show that $M$ is Fredholm with index 0 . We will do this by multiplying $M$ by manifestly invertible maps until we reduce the problem of showing that $M$ is Fredholm to the problem of showing that a certain map on $W$ is Fredholm. To begin first multiply $M$ on the right by the $p \times p$ diagonal matrix with $(k, k)$ entry $\left(\begin{array}{cc}1 & 0 \\ 0 & -\varepsilon_{k}\end{array}\right)$. Then transform the result by a similarity with the $p \times p$ diagonal matrix that has $T\left(n, n_{k}\right)$ as its $(k, k)$ entry. Introduce the notation: $s_{j}=-T\left(n, n_{j}\right) \varepsilon_{j} T\left(n_{j}, n\right)$. Then the result, which we illustrate in the typical case $p=3$, is:

$$
\left[\begin{array}{ccc}
Q_{-} s_{1}+Q_{+} & -Q_{+}\left(s_{2}-1\right) & -Q_{+}\left(s_{3}-1\right) \\
Q_{-}\left(s_{1}-1\right) & Q_{-} s_{2}+Q_{+} & -Q_{+}\left(s_{3}-1\right) \\
Q_{-}\left(s_{1}-1\right) & Q_{-}\left(s_{2}-1\right) & Q_{-} s_{3}+Q_{+}
\end{array}\right]
$$

Now multiply this matrix on the left first by:

$$
\left[\begin{array}{ccc}
1 & -1 & 0 \\
0 & 1 & -1 \\
0 & 0 & 1
\end{array}\right]
$$


and then in succession by:

and

$$
\left[\begin{array}{ccc}
1 & 0 & 0 \\
0 & 1 & 0 \\
-Q_{-}\left(s_{1}-1\right) & 0 & 1
\end{array}\right]
$$

$$
\left[\begin{array}{ccc}
1 & 0 & 0 \\
0 & 1 & 0 \\
0 & -Q_{-}\left(s_{1} s_{2}-1\right) & 1
\end{array}\right] .
$$

One finds the upper triangular matrix:

$$
\left[\begin{array}{ccc}
1 & -s_{1} & 0 \\
0 & 1 & -s_{2} \\
0 & 0 & Q_{-}\left(s_{1} s_{2} s_{3}\right)+Q_{+}
\end{array}\right] .
$$

Thus $M$ will be Fredholm with index 0 if and only if $Q_{-}\left(s_{1} s_{2} s_{3}\right)+Q_{+}$is Fredholm with index 0 . The precisely analogous calculation for general $p$ shows that one must consider $Q_{-}\left(s_{1} s_{2} \cdots s_{p}\right)+Q_{+}$. Recall that in Sect. 2 we introduced the notation $S=\left(\begin{array}{ll}A(S) & B(S) \\ C(S) & D(S)\end{array}\right)$ for the matrix of a linear map $S$ on $W$ relative to an isotropic splitting $W_{+}+W_{-}$of $W$. The matrix of $Q_{-}\left(s_{1} s_{2} \cdots s_{p}\right)+Q_{+}$relative to the isotropic splitting $W_{+}+W_{-}$is:

$$
\left(\begin{array}{cc}
1 & 0 \\
C\left(s_{1} \cdots s_{p}\right) & D\left(s_{1} \cdots s_{p}\right)
\end{array}\right) .
$$

Our problem then is to show that $D\left(s_{1} \cdots s_{p}\right)$ is Fredholm with index 0 . The matrix of $s_{1} \cdots s_{p}$ relative to the isotropic splitting of $W$ is obtained by multiplying together the matrices for each of the factors $s_{j}$. Thus $D\left(s_{1} s_{2} \cdots s_{p}\right)=D\left(s_{1}\right) D\left(s_{2}\right) \cdots D\left(s_{p}\right)+$ terms each of which contains at least one factor $B\left(s_{j}\right)$ or $C\left(s_{j}\right)$ for $j=1,2, \ldots, p$. We will now show that each $D\left(s_{j}\right)$ is Fredholm with index 0 and each $B\left(s_{j}\right)$ and $C\left(s_{j}\right)$ is in the Schmidt class. From this it follows directly that $D\left(s_{1}\right) D\left(s_{2}\right) \cdots D\left(s_{p}\right)$ is Fredholm with index 0 and thus so is $D\left(s_{1} s_{2} \cdots s_{p}\right)$ since it is a compact perturbation of this product.

It is easy to see that $\varepsilon_{j}$ (and hence also $s_{j}$ ) is a complex orthogonal on $W$. If we show that the commutator of $\varepsilon_{j}$ and $Q$ is in the Schmidt class, then $\varepsilon_{j}$ (and hence also $s_{j}$ ) is in $O_{\text {res }}(W)$. It follows that $D\left(\varepsilon_{j}\right)$ (and hence also $D\left(s_{j}\right)$ ) is Fredholm with index 0 (see [CP] or [PS]). In the Fourier transform variable $z$, the map $\varepsilon_{j}$ is a singular convolution operator with a principal value singularity $\left(z^{\prime}-z\right)^{-1}$ on the diagonal. The behavior of the kernel for $\varepsilon_{j} Q-Q \varepsilon_{j}$ on the diagonal is thus the same as $\left(Q\left(z^{\prime}\right)-Q(z)\right) /\left(z^{\prime}-z^{\prime}\right)$. The formula above for the multiplication operators $Q_{ \pm}(z)$ shows that $Q(z)$ is a smooth function of $z \in S^{1}$. Thus the commutator of $\varepsilon_{j}$ and $Q$ has a continuous square integrable kernel in the Fourier transform variable and consequently is in the Schmidt class. This finishes the proof that $L_{\Gamma}$ is Fredholm with index 0 when (A) and (B) are satisfied. This is certainly not the simplest derivation of this result, but the calculations we've done are of interest for other 
reasons as well. At the level of determinant bundles we've shown that the problem of defining a determinant for $L_{\Gamma}$ can be "reduced" to the problem of defining a determinant for $D\left(s_{1} s_{2} \cdots s_{p}\right)$. In [PT] and [P] it is shown that the correct choice of a determinant for $D\left(s_{1} s_{2} \cdots s_{p}\right)$ (the choice that gives the square of the correlation function) has an elegant characterization in terms of a $Z / 2 Z$ homomorphic lift $s_{j} \rightarrow \sigma_{j} \in \hat{\mathrm{S}} \operatorname{pin}(W)$. The calculations we've just done might be of some help in trying to give a more direct characterization of the appropriate trivialization for the determinant bundle over $\Gamma \rightarrow L_{\Gamma}$. We hope to pursue this in another place. For the present we turn to the final topic of this paper: the identification of the Pfaffian bundle over $\Gamma \rightarrow L_{\Gamma}$ with a sub-bundle of the Pfaffian bundle over the isotropic Grassmannian, $G r_{\text {iso }}$.

\section{Two Pfaffian Bundles}

Recall that $n$ and $N$ are integers chosen so that $n<n_{1}$ and $n_{p}<N$. We will now introduce truncated versions of $L_{\Gamma}$ defined by boundary conditions at $n$ and $N$. We first define $L_{+}\left(W_{-}\right)$which we also denote by $L_{+}$for brevity. The domain $\mathscr{D}_{+}$ of $L_{+}$consists of functions $F \in H_{\mathbf{C}}$ with $\partial_{N} F \in W_{-}$and with $F_{j}(k, l)=0$ for $l>N$ unless $l=N-\frac{1}{2}$ and $j=4$. Let $P_{+}$denote the projection on the subspace of $H_{\mathrm{C}}$ defined by the condition $F(k, l)=0$ for $l<N$. We define:

$$
L_{+}=P_{+} L_{\Gamma} \mid \mathscr{D}_{+} \text {. }
$$

In a similar fashion we define $L_{-}$as follows. Let $\mathscr{D}_{-}$consist of those functions $F \in H_{\mathrm{C}}$ with $\partial_{n} F \in W_{+}$and $F_{j}(k, l)=0$ if $l<n$ unless $l=n+\frac{1}{2}$ and $j=3$. Let $P_{-}$ denote the orthogonal projection on the subspace of $H_{\mathrm{C}}$ defined by the condition $F(k, l)=0$ for $l>n$. We define:

$$
L_{-}=P_{-} L_{\Gamma} \mid \mathscr{D}_{-} \cdot
$$

Finally we introduce an operator $L\left(W_{+}, W_{-}\right)$obtained from $L_{\Gamma}$ by imposing boundary conditions at $n$ and $N$. Let $P_{0}:=I-P_{+}-P_{-}$and define $\mathscr{D}_{0}=$ $\left\{F \in H_{\mathbf{C}}: \partial_{n} F \in W_{+}, \partial_{N} F \in W_{-}\right.$, and $F_{j}(k, l)=0$ if $l>N$ or $l<n$ unless $l=N-\frac{1}{2}$ and $j=4$ or $l=n+\frac{1}{2}$ and $\left.j=3\right\}$. We define:

$$
L\left(W_{+}, W_{-}\right)=P_{0} L_{\Gamma} \mid \mathscr{D}_{0} .
$$

Our main interest is in $L\left(W_{+}, W_{-}\right)$. The kernel and cokernel of $L_{\Gamma}$ can be identified with the kernel and cokernel of $L\left(W_{+}, W_{-}\right)$as we demonstrate in the following proposition:

Proposition. The map $\operatorname{ker}\left(L_{\Gamma}\right) \in F \rightarrow F \mid \mathscr{D}_{0}$ is an isomorphism of the kernel of $L_{\Gamma}$ with the kernel of $L\left(W_{+}, W_{-}\right)$. The natural inclusion $R\left(P_{0}\right) \rightarrow H_{\mathrm{C}}$ of the range of $P_{0}$ in $H_{\mathrm{C}}$ induces an isomorphism $\operatorname{coker}\left(L\left(W_{+}, W_{-}\right)\right) \rightarrow \operatorname{coker}\left(L_{\Gamma}\right)$.

Proof. Suppose $F \in \operatorname{ker}\left(L_{\Gamma}\right)$. Then the transfer matrix equation (T) shows that $T^{m} \partial_{n} F=\partial_{n-m} F$, and it follows that $\partial_{n} F$ must be in $W_{+}$if $F$ is to be square summable on $Z_{1 / 2}^{2}$. For similar reasons we must have $\partial_{N} F \in W_{-}$. Thus if $F \in \operatorname{ker}\left(L_{\Gamma}\right)$ then $F$ has a natural restriction, $F \mid \mathscr{D}_{0}$, to the domain $\mathscr{D}_{0}$. Furthermore, one can easily check that $L_{\Gamma} F$ and $L\left(W_{+}, W_{-}\right)\left(F \mid \mathscr{D}_{0}\right)$ have the same values in the range 
of $P_{0}$. Thus:

$$
L\left(W_{+}, W_{-}\right)\left(F \mid \mathscr{D}_{0}\right)=P_{0} L_{\Gamma} F=0,
$$

and it follows that $F \mid \mathscr{D}_{0}$ is in $\operatorname{ker}\left(L\left(W_{+}, W_{-}\right)\right)$. To see that $\operatorname{ker}\left(L_{\Gamma}\right) \in F \rightarrow F \mid \mathscr{D}_{0} \in$ $\operatorname{ker}\left(L\left(W_{+}, W_{-}\right)\right)$is injective suppose that $F \mid \mathscr{D}_{0}=0$. Then equation (T) for $L_{\Gamma} F=0$ implies that $F=0$ since $\partial_{n} F=\partial_{N} F=0$. To see that the map in question is surjective observe that (T) shows one how to extend any element $f \in \mathscr{D}_{0}$ in the kernel of $L\left(W_{+}, W_{-}\right)$to an element $F \in \operatorname{ker}\left(L_{\Gamma}\right)$. The observation is again simply that since $\partial_{n} f \in W_{+}$and $\partial_{N} f \in W_{-}$one may apply appropriate powers of the transfer matrix $T$ to those boundary values to produce square summable functions on $Z_{1 / 2}^{2}$.

Next we turn to the cokernel result. Recall that for a map $A$ on $H_{\mathrm{C}}$, $\operatorname{coker}(A)$ is the vector space quotient of $H_{\mathrm{C}}$ by $R(A)$, the range of $A$. We first show that if $G \in R_{0}:=$ range of $L\left(W_{+}, W_{-}\right)$, then $G$ is also in the range of $L_{\Gamma}$. Suppose then that there is a vector $F \in \mathscr{D}_{0}$ such that:

$$
P_{0} L_{\Gamma} F=G
$$

Because $\partial_{n} F \in W_{+}$and $\partial_{N} F \in W_{-}$, we may use (T) to extend $F$ to a square summable solution, $F^{\prime}$, to:

$$
L_{\Gamma} F^{\prime}=G
$$

The natural inclusion $R\left(P_{0}\right) \ni G \rightarrow G \in H_{\mathrm{C}}$ thus induces a map from $\operatorname{coker}\left(L\left(W_{+}, W_{-}\right)\right)$ to coker $\left(L_{\Gamma}\right)$. We wish to show that this induced map is bijective. Suppose that $G \in R\left(P_{0}\right)$ maps into 0 in coker $\left(L_{\Gamma}\right)$ (i.e., $G$ is in the range of $\left.L_{\Gamma}\right)$. Then there is $F \in H_{\mathrm{C}}$ such that $L_{\Gamma} F=G$. But (T) implies that for $F$ to be in $H_{\mathrm{C}}$ we must have $\partial_{n} F \in W_{+}$and $\partial_{N} F \in W_{-}$. Thus we can restrict $F$ to $\mathscr{D}_{0}$ and as may be easily checked: $L\left(W_{+}, W_{-}\right)\left(F \mid \mathscr{D}_{0}\right)=P_{0} L_{\Gamma} F=P_{0} G=G$. Thus $G \in R_{0}:=\operatorname{range}$ of $L\left(W_{+}, W_{-}\right)$. This shows the inclusion is injective. To see that it is surjective we make use of the fact that our assumptions (A) and (B) imply that both $L_{+}$and $L_{-}$are invertible. To see this suppose that $G \in P_{+} H_{\mathrm{C}}$ and $F \in H_{\mathrm{C}}$ is the solution to $L_{0} F=G$. Then the transfer relation (T) for $L_{0} F=G$ shows that we must have $\partial_{N} F \in W_{+}$. But then as above: $L_{+}\left(F \mid \mathscr{D}_{+}\right)=P_{+} L_{\Gamma}\left(F \mid \mathscr{D}_{+}\right)=P_{+} L_{\Gamma} F=P_{+} G=G$. In a similar fashion one can show $L_{-}$is invertible. Now suppose that $G \in H_{\mathbf{C}}$. To show that the inclusion above is surjective we must find an element $G^{\prime}$ in the range of $P_{0}$ so that $G-G^{\prime}$ is in the range of $L_{\Gamma}$. Here is one way to do this. Let $F_{ \pm}$denote the solutions in $\mathscr{D}_{ \pm}$to $L_{ \pm} F_{ \pm}=P_{ \pm} G$. One may also regard $F_{ \pm}$as elements in $H_{\mathrm{C}}$ in a natural way (i.e., so that the projection of $F_{ \pm}$on the orthogonal complement of $\mathscr{D}_{ \pm}$is 0 ). Regarding $F_{ \pm}$as an element of $H_{\mathrm{C}}$ in this fashion one finds:

$$
L_{\Gamma} F_{ \pm}=P_{ \pm} G+\Delta_{ \pm} .
$$

The terms $\Delta_{ \pm}$are both in the range of $P_{0}$. Thus we find that $G$ and $G^{\prime}:=P_{0} G-$ $\Delta_{+}-\Delta_{-} \in R\left(P_{0}\right)$ differ by $P_{+} G+P_{-} G+\Delta_{+}+\Delta_{-}$which is $L_{\Gamma}\left(F_{+}+F_{-}\right)$. This finishes the proof of the proposition. QED.

This proposition suggests an alternative proof that the maps $L_{\Gamma}$ are Fredholm with index 0 that we now sketch. Without much difficulty one may use the techniques in the proof of the proposition to show that if $L\left(W_{+}, W_{-}\right)$can be made invertible by a finite rank perturbation then the same is true for $L_{\Gamma}$. 
Consider now the transfer matrix, $T_{\Gamma}(n, N)$, for $L\left(W_{+}, W_{-}\right)$which takes one from level $N$ to level $n$. This was calculated in Sect. 3. One has:

$$
T_{\Gamma}(n, N)=(-1)^{p} T\left(n, n_{1}\right) \varepsilon_{m_{1}} T\left(n_{1}, n_{2}\right) \varepsilon_{m_{2}} \cdots T\left(n_{p-1}, n_{p}\right) \varepsilon_{m_{p}} T\left(n_{p}, N\right)
$$

where $T(a, b):=T^{b-a}$. If we now introduce (as we did in Sect. 3):

$$
s_{j}:=-T\left(n, n_{j}\right) \varepsilon_{m_{j}} T\left(n_{j}, n\right)
$$

then we can rewrite this as:

$$
T_{\Gamma}(n, N)=s_{1} s_{2} \cdots s_{p} T(n, N) .
$$

The calculations at the end of Sect. 3 show that $T_{\Gamma}(n, N) \in O_{\text {res }}(W)$ relative to the isotropic splitting $W=W_{+}+W_{-}$.

Suppose now that $G \in R\left(P_{0}\right)$ and that one attempts to solve $L\left(W_{+}, W_{-}\right) F=G$ for $F$ by using the transfer relation (T). One is confronted with:

$$
\partial_{n} F=T_{\Gamma}(n, N) \partial_{N} F+\text { inhomogeneous terms. }
$$

For this equation to uniquely determine the boundary values $\partial_{n} F$ and $\partial_{N} F$ it is necessary and sufficient that the subspace $T_{\Gamma}(n, N) W_{-}$be transverse to $W_{+}$. This may not be true but because $T_{\Gamma}(n, N) \in O_{\text {res }}(W)$ it can fail only by a finite dimensional amount in the following sense. There exists a finite rank perturbation of the identity $\gamma \in O_{\text {res }}(W)$ such that the subspace $\gamma T_{\Gamma}(n, N) W_{-}$is transverse to $W_{+}$. Note that when the dimension of $T_{\Gamma}(n, N) W_{-} \cap W_{+}$is odd $\gamma$ will include a complex orthogonal reflection as well as a map $\gamma_{c}$ from Sect. 2. Thus $T_{\Gamma}(n, N) W_{-}$is transverse to $U:=\gamma^{-1} W_{+}$. It is natural then to introduce the operator $L\left(U, W_{-}\right)$which is the same as $L\left(W_{+}, W_{-}\right)$except that the condition that defines the domain at level $N$ is $\partial_{N} F \in U$ rather than $\partial_{N} F \in W_{+}$. It is not hard to use the transfer relation (T) to see that this alteration in the boundary conditions produces an invertible operator. To obtain a finite rank perturbation of $L\left(W_{+}, W_{-}\right)$which is invertible, one may adjust the domain of $L\left(U, W_{-}\right)$to agree with that of $L\left(W_{+}, W_{-}\right)$by composing on the right with the map which multiplies $\partial_{N} F$ by $\gamma^{-1}$ but acts as the identity on $\partial_{N} H_{\mathrm{C}}^{\perp}$. This finishes our sketch of an alternative proof that $L_{\Gamma}$ is Fredholm with index 0 . This proof is a translation of an idea in Witten [W].

In order to state that principal result of this paper we now make our final assumption regarding the parameters in $L_{\Gamma}$. It is:

$$
T_{\Gamma}(n, N) \in S O_{\text {res }}(W) .
$$

To orient the reader we note that $(\mathrm{C})$ will always be true for the Ising model below the critical temperature. Above the critical temperature $(\mathrm{C})$ is true when $p$ is even but false when $p$ is odd. Our assumption (C) is equivalent to supposing that:

$$
L_{\Gamma} \in S k_{0}\left(H_{\mathbf{C}}\right) \text {. }
$$

We know that $L_{\Gamma}$ is Fredholm with index 0 . To see that it is in $S k_{0}\left(H_{\mathrm{C}}\right)$ we need to know that $\operatorname{ker}\left(L_{\Gamma}\right)$ is even dimensional. To see that this follows from $(\mathrm{C})$ suppose that $F \in \mathscr{D}_{0}$ is in the kernel of $L\left(W_{+}, W_{-}\right)$. The transfer relation (T) for $L\left(W_{+}, W_{-}\right)$ $F=0$ shows that:

$$
T_{\Gamma}(n, N) \partial_{N} F=\partial_{n} F
$$




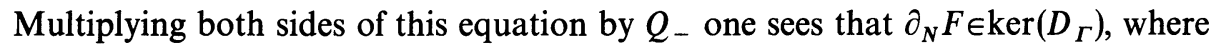
$D_{\Gamma}$ is the $D$ matrix element of $T_{\Gamma}(n, N)$ relative to the isotropic splitting $W=W_{+}+W_{-}$. Simple arguments like those used in the proof of the proposition in this section show that the map $\operatorname{ker}\left(L\left(W_{+}, W_{-}\right)\right) \in F \rightarrow \partial_{N} F \in \operatorname{ker}\left(D_{\Gamma}\right)$ is an isomorphism. The dimension of $\operatorname{ker}\left(D_{\Gamma}\right)$ is even if and only if $(C)$ is true [CP]. Thus when $(C)$ is true the dimension of $\operatorname{ker}\left(L\left(W_{+}, W_{-}\right)\right)$is even. The proposition of this section then implies that $\left(C^{\prime}\right)$ is true as well.

We are now prepared to state the main result of this paper. Before we do this we recall the significance of the assumptions (A), (B), and (C). The first assumption guarantees that the translation invariant version of the difference operators we consider is invertible. The second assumption guarantees that the relation between the difference operator and its associated transfer matrix is non-critical. The final assumption is that the "parity" index for the family of operators we consider is 0 .

Theorem. Suppose $(A),(B)$ and $(C)$ are true. Then the Pfaffian bundle over $\Gamma \rightarrow L_{\Gamma} \in S k_{0}\left(H_{\mathrm{C}}\right)$ and the Pfaffian bundle over $\Gamma \dot{\rightarrow} T_{\Gamma}(n, N) W_{-} \in G r_{\text {iso }}$ are isomorphic.

Proof. We will show that the fibers over these two bundles can be naturally identified. For dramatic effect we have stated this result in terms of the Pfaffian bundle over the isotropic Grassmannian. Recall however that this pulls back to the spin bundle over the restricted orthogonal group under the map $T \rightarrow T W_{-}$. We will deal with the spin bundle over $\Gamma \rightarrow T_{\Gamma}(n, N)$ rather than the Pfaffian bundle.

We begin by explaining a slightly different way of looking at the trivializations for the Pfaffian bundle over $S k_{0}\left(H_{\mathrm{C}}\right)$ and the spin bundle over $S O_{\text {res }}(W)$ that were constructed in Sects. 1 and 2. Suppose $L=L_{\Gamma}$ is a fixed element in $S k_{0}\left(H_{\mathrm{C}}\right)$. One may define a trivialization of the Pfaffian bundle in a neighborhood of $L$ by choosing a skew symmetric map $F: \operatorname{ker}(L) \rightarrow \operatorname{Cok}(L)$, where $\operatorname{Cok}(L)$ is a subspace of $H_{\mathrm{C}}$ transverse to $R(L)$ and which is non-degenerate with respect to the bilinear form on $H_{\mathbf{C}}$. The map $F$ induces a map $\hat{F}: \operatorname{ker}(L) \rightarrow \operatorname{coker}(L)$, where $\operatorname{coker}(L)$ is $H_{\mathrm{C}} \bmod R(L)$. In fact one can reconstruct $F$ from $\hat{F}$ and the choice of the subspace $\operatorname{Cok}(L)$ as we now demonstrate. Recall from Sect. 1 that $\operatorname{coker}(L)$ is naturally identified with the dual space $\operatorname{ker}(L)^{*}$. Choose an isomorphism $\hat{F}: \operatorname{ker}(L) \rightarrow \operatorname{ker}(L)^{*}$ which is skew symmetric in the sense that the natural dual map $\hat{F}^{\tau}: \operatorname{ker}(L) \rightarrow \operatorname{ker}(L)^{*}$ is equal to $-\hat{F}$. Now choose a subspace $\operatorname{Cok}(L)$ transverse to $R(L)$ and non-degenerate with respect to the bilinear form. There is a natural map from $\operatorname{coker}(L)$ to $\operatorname{Cok}(L)$ given by $x+R(L) \rightarrow x^{\prime}$, where $x^{\prime}+R(L)$ is the unique representative for $x+R(L)$ with $x^{\prime} \in \operatorname{Cok}(L)$. If we now extend $\hat{F}$ to a map $F$ from $\operatorname{ker}(L)$ to $\operatorname{Cok}(L)$ through the identifications $\operatorname{ker}(L)^{*} \simeq \operatorname{coker}(L) \simeq \operatorname{Cok}(L)$ then it is not hard to check that $F$ is skew symmetric with respect to the bilinear form on $H_{\mathrm{C}}$. Thus $F$ is suitable to define a trivialization of the Pfaffian bundle in a neighborhood of $L$.

Something similar works for the spin group. Suppose that $\left(\begin{array}{ll}A & B \\ C & D\end{array}\right)$ is the matrix of the complex orthogonal $T:=T_{\Gamma}(n, N)$ with respect to the isotropic splitting $W_{+}+W_{-}$. We claim that the space $B \operatorname{ker}(D)$ is naturally isomorphic to the dual of the space $\operatorname{coker}(D)$. We first observe that $B \operatorname{ker}(D)=\operatorname{ker}\left(D^{\tau}\right)$. To see this note 
that $B^{\tau} D+D^{\tau} B=0$ since $T$ is complex orthogonal. Thus $B \operatorname{ker}(D) \subseteq \operatorname{ker}\left(D^{\tau}\right)$. But again since $T$ is complex orthogonal $A^{\tau} D+C^{\tau} B=I$ so that $B$ is non-singular when restricted to $\operatorname{ker}(D)$. However $D$ has index 0 so that $\operatorname{dim}(\operatorname{ker}(D))=\operatorname{dim}\left(\operatorname{ker}\left(D^{*}\right)\right)=$ $\operatorname{dim}\left(\operatorname{ker}\left(D^{\tau}\right)\right)$. Thus $B: \operatorname{ker}(D) \rightarrow \operatorname{ker}\left(D^{\tau}\right)$ is an isomorphism. Now let $(\cdot, \cdot)$ denote the distinguished bilinear form on $W$ and consider the map $\operatorname{ker}\left(D^{c}\right) \ni x \rightarrow(x, \cdot)$. The linear functional $(x, \cdot)$ is well define on $\operatorname{coker}(D)$ if $x \in \operatorname{ker}\left(D^{\tau}\right)$. To see this suppose that $x \in \operatorname{ker}\left(D^{\tau}\right)$ and $y \in W_{-}$then: $(x, D y)=\left(D^{\tau} x, y\right)=0$. The map $\operatorname{ker}\left(D^{\tau}\right) \ni x \rightarrow(x, \cdot) \in$ $\operatorname{coker}(D)^{*}$ is injective since any $x$ which maps to zero is complex orthogonal to all of $W$. But we've seen that the dimension of $\operatorname{ker}\left(D^{\tau}\right)$ and the dimension of $\operatorname{coker}(D)^{*}$ are the same so the map in question must be an isomorphism.

We may recast the data needed for the trivialization of the spin bundle in a neighborhood of $T$ as follows. First choose an isomorphism $\hat{c}: \operatorname{ker}\left(D^{\tau}\right) \rightarrow \operatorname{ker}\left(D^{\tau}\right)^{*}$ which is skew symmetric in the sense that the natural dual map $\hat{c}^{\tau}$ is equal to $-\hat{c}$. Second choose a subspace $\operatorname{Cok}(D)$ which is transverse to $R(D)$. The bilinear form on $W$ gives a non-degenerate pairing between $W_{+}$and $W_{-}$. The direct sum decomposition $R(D)+\operatorname{Cok}(D)$ of $W_{-}$is reflected in the direct sum decomposition $R(D)^{\perp}+\operatorname{Cok}(D)^{\perp}$ of $W_{+}$, where $X^{\perp}$ is the annihilator of $X \subseteq W_{-}$in $W_{+} \simeq W_{-}^{*}$. We have seen that $R(D)^{\perp}=\operatorname{ker}\left(D^{\tau}\right)$. Thus $\operatorname{ker}\left(D^{\tau}\right)+\operatorname{Cok}(D)^{\perp}$ is a direct sum decomposition of $W_{+}$. Extend $\hat{c}$ to a map $c$ from $\operatorname{ker}\left(D^{\tau}\right)$ to $\operatorname{Cok}(D)$ via the identifications $\operatorname{ker}\left(D^{\tau}\right)^{*} \simeq \operatorname{coker}(D) \simeq \operatorname{Cok}(D)$. Then extend $c$ to a map from $W_{+}$ to $W_{\text {- }}$ by setting $c(x)=0$ for $x \in \operatorname{Cok}(D)^{\perp}$. One may check that the resulting map (which we continue to denote by $c$ ) is skew symmetric and that $D+c B$ is invertible (it is Fredholm, has index 0 , and no kernel). Thus $c$ is suitable to define a trivialization of the spin bundle $\hat{\operatorname{Spin}}(W) \rightarrow S O_{\text {res }}(W)$ in a neighborhood of $T \in S O_{\text {res }}(W)$.

Suppose that $L:=L_{\Gamma}$ for some $\Gamma$ and that $\hat{F}: \operatorname{ker}(L) \rightarrow \operatorname{ker}(L)^{*}$ is a skew symmetric isomorphism and that $\operatorname{Cok}(L)$ is a subspace transverse to $R(L)$ which is non-degenerate with respect to the bilinear form on $H_{\mathrm{C}}$. One then has a trivialization $U_{F} \times C^{*} \ni(L, \lambda) \rightarrow \lambda \sigma_{F}(L)$ for $P f$ at $L$. Suppose $f \in \operatorname{ker}(L)$ and consider the map $f \rightarrow \partial_{n} f$. Since $\partial_{n} f \in W_{+}, \partial_{N} f \in W_{-}$and $T \partial_{N} f=\partial_{n} f$, it follows that $D \partial_{N} f=0$ and $\partial_{n} f=B \partial_{N} f$. It is easy to check that the map $\operatorname{ker}(L) \in f \rightarrow \partial_{n} f$ extends to an isomorphism, $i$, of $\operatorname{ker}(L)$ with $B \operatorname{ker}(D)=\operatorname{ker}\left(D^{\tau}\right)$. Now define $\hat{c}=i^{-\tau} \hat{F} i^{-1}$, where $i^{-\tau}$ is the map dual to $i^{-1}$. The map $\hat{c}$ is then a skew symmetric isomorphism from $\operatorname{ker}\left(D^{\tau}\right)$ to $\operatorname{ker}\left(D^{\tau}\right)^{*}$. As above the choice of a subspace $\operatorname{Cok}(D)$ transverse to $R(D)$ in $W_{-}$is all the additional information needed to define a map $c: W_{+} \rightarrow W_{-}$suitable to determine a trivialization $\sigma_{c}$ of the spin bundle in a neighborhood of $T$ (see Sect. 2).

We now identify the fiber of the Pfaffian bundle at $L$ with the fiber of the spin bundle at $T$ by mapping $\lambda \sigma_{F}(L)$ to $\lambda \sigma_{c}(T)$. We will now verify that this identification does not depend on which map $\hat{F}$ is initially chosen nor does it depend on the choice of the subspaces $\operatorname{Cok}(L)$ and $\operatorname{Cok}(D)$. To begin suppose that $\operatorname{Cok}(L)$ and $\operatorname{Cok}(D)$ are fixed and that $\hat{F}_{1}$ and $\hat{F}_{2}$ are two skew isomorphisms from $\operatorname{ker}(L)$ to $\operatorname{ker}(L)^{*}$. To see that the isomorphism we've defined does not depend on $\hat{F}_{j}$ we must show that:

$$
\frac{\sigma_{F_{2}}(L)}{\sigma_{F_{1}}(L)}=\frac{\sigma_{c_{2}}(T)}{\sigma_{c_{1}}(T)}
$$


where $c_{j}$ is derived from $\hat{F}_{j}$ as above. In Sects 1 and 2 we saw that:

and

$$
\frac{\sigma_{F_{2}}(L)}{\sigma_{F_{1}}(L)}=P f\left(\left(L+F_{1}\right)\left(L+F_{2}\right)^{-1}\right)
$$

$$
\frac{\sigma_{c_{2}}(T)}{\sigma_{c_{1}}(T)}=P f\left(\left(D+c_{1} B\right)\left(D+c_{2} B\right)^{-1}\right) .
$$

We first consider the relative Pfaffian $\operatorname{Pf}\left(\left(L+F_{1}\right)\left(L+F_{2}\right)^{-1}\right)$. The space $H_{\mathrm{C}}$ is the direct sum $\operatorname{ker}(L)+\operatorname{Cok}(L)^{\perp}$. Since $F_{j} \mid \operatorname{Cok}(L)^{\perp}=0$ it is easy to see that $\left(L+F_{1}\right)\left(L+F_{2}\right)^{-1}=\hat{F}_{1} \hat{F}_{2}^{-1} \oplus I$ relative to this direct sum decomposition. Thus $\operatorname{det}\left(\left(L+F_{1}\right)\left(L+F_{2}\right)^{-1}\right)=\operatorname{det}\left(\hat{F}_{1} \hat{F}_{2}^{-1}\right)$.

In a similar fashion consider the direct sum decomposition $R(D)+\operatorname{Cok}(D)$ for $W_{-}$. Suppose $x_{1} \in R(D)$ and $x_{2} \in \operatorname{Cok}(D)$. Then: $\left(D+c_{1} B\right)\left(D+c_{2} B\right)^{-1}\left(x_{1}+x_{2}\right)=$ $\left(D+c_{1} B\right)\left(D+c_{2} B\right)^{-1} x_{1}+\left(c_{1} B\right)\left(c_{2} B\right)^{-1} x_{2}$, where $c_{j} B$ is regarded as an isomorphism of $\operatorname{ker}(D)$ with $\operatorname{Cok}(D)$ in the last term. But $\left(c_{1} B\right)\left(c_{2} B\right)^{-1} x_{2} \in \operatorname{Cok}(D)$ and $\left(D+c_{1} B\right)\left(D+c_{2} B\right)^{-1} x_{1}=x_{1}+\left(c_{1}-c_{2}\right) B\left(D+c_{2} B\right)^{-1} x_{1}$. The second term in this sum is in $\operatorname{Cok}(D)$. Thus we find the matrix of $\left(D+c_{1} B\right)\left(D+c_{2} B\right)^{-1}$ relative to the direct sum $R(D)+\operatorname{Cok}(D)$ is:

$$
\left(\begin{array}{cc}
I & 0 \\
* & \left(c_{1} B\right)\left(c_{2} B\right)^{-1}
\end{array}\right) .
$$

Hence $\operatorname{det}\left(\left(D+c_{1} B\right)\left(D+c_{2} B\right)^{-1}\right)=\operatorname{det}\left(\left(c_{1} B\right)\left(c_{2} B\right)^{-1}\right)=\operatorname{det}\left(\hat{c}_{1} \hat{c}_{2}^{-1}\right)=\operatorname{det}\left(\hat{F}_{1} \hat{F}_{2}^{-1}\right)$. We have confirmed the square of the equality we desire. To obtain equality at the level of Pfaffians observe that the set of skew symmetric isomorphisms from $\operatorname{ker}(L)$ to $\operatorname{ker}(L)^{*}$ is path connected. Thus we can find a continuous path $[0,1] \in t \rightarrow \hat{F}(t)$ such that $\hat{F}(0)=F_{2}$ and $\hat{F}(1)=F_{1}$. Let $F(t)$ and $c(t)$ denote the maps derived from $\hat{F}(t)$ as above. The Pfaffians $P f\left((L+F(t))\left(L+F_{2}\right)^{-1}\right)$ and $P f\left((D+c(t) B)\left(D+c_{2} B\right)^{-1}\right)$ are continuous non-zero functions of $t$ which are both equal to 1 at $t=0$ and have equal squares for $t \in[0,1]$. Thus they must be equal for all $t$, in particular for $t=1$. This finishes the proof that the isomorphism of fibers does not depend on the choice of $\hat{F}$.

Next suppose that $\hat{F}$ and $\operatorname{Cok}(D)$ are fixed and that $\operatorname{Cok}_{1}(L)$ and $\operatorname{Cok}_{2}(L)$ are two non-degenerate subspaces transverse to $R(L)$. The extensions $c_{1}$ and $c_{2}$ are equal so that the relative Pfaffian $P f\left(\left(D+c_{1} B\right)\left(D+c_{2} B\right)^{-1}\right)$ is equal to 1 . The maps $F_{1}$ and $F_{2}$ are not necessarily equal but we do have $F_{1}(x)-F_{2}(x) \in R(L)$ for $x \in \operatorname{ker}(L)$. Since $\operatorname{Cok}_{1}(L)$ and $\operatorname{Cok}_{2}(L)$ are both transverse to $R(L)$ it follows that the projection, $p r$, of $\operatorname{Cok}_{1}(L)$ on $\operatorname{Cok}_{2}(L)$ along $R(L)$ is an isomorphism. Suppose $x=x_{1}+x_{2}$ with $x_{1} \in \operatorname{Cok}_{1}(L)$ and $x_{2} \in R(L)$. Define $M x=\operatorname{pr}\left(x_{1}\right)+x_{2}$. Observe that $M\left(x_{1}+x_{2}\right)=x_{1}+\left(\operatorname{pr}\left(x_{1}\right)-x_{1}\right)+x_{2}$, where $\operatorname{pr}\left(x_{1}\right)-x_{1} \in R(L)$. Thus the matrix of $M$ relative to the splitting $\operatorname{Cok}_{1}(L)+R(L)$ is $\left(\begin{array}{ll}I & 0 \\ * & I\end{array}\right)$ and it follows that $\operatorname{det}(M)=1$.

Now we suppose $x=x_{1}+x_{2}$ with $x_{1} \in \operatorname{Cok}_{2}(L)$ and $x_{2} \in R(L)$ and calculate:

$$
\begin{aligned}
M\left(L+F_{1}\right)\left(L+F_{2}\right)^{-1}\left(x_{1}+x_{2}\right) & =M\left(L+F_{1}\right)\left(F_{2}^{-1} x_{1}+\left(L+F_{2}\right)^{-1} x_{2}\right) \\
& =M\left(F_{1} F_{2}^{-1} x_{1}+x_{2}+F_{1}\left(L+F_{2}\right)^{-1} x_{2}\right) .
\end{aligned}
$$


Observe that when restricted to $\operatorname{ker}(L)$ we have $M F_{1}=F_{2}$, since for $y \in \operatorname{ker}(L)$ the difference $F_{1}(y)-F_{2}(y) \in R(L)$ it follows that $\operatorname{pr}\left(F_{1}(y)\right)=F_{2}(y)$. Thus $M F_{1} F_{2}^{-1} x_{1}=$ $F_{2} F_{2}^{-1} x_{1}=x_{1}$. We have then:

$$
M\left(L+F_{1}\right)\left(L+F_{2}\right)^{-1}\left(x_{1}+x_{2}\right)=x_{1}+x_{2}+M F_{1}\left(L+F_{2}\right)^{-1} x_{2},
$$

where $M F_{1}\left(L+F_{2}\right)^{-1} x_{2} \in \operatorname{Cok}_{2}(L)$. It follows that the matrix of $M\left(L+F_{1}\right)$ $\left(L+F_{2}\right)^{-1}$ relative to the splitting $\operatorname{Cok}_{2}(L)+R(L)$ is $\left(\begin{array}{ll}I & * \\ 0 & I\end{array}\right)$ so that the determinant of this operator is clearly 1 . Since $\operatorname{det}(M)=1$ we also have:

$$
\operatorname{det}\left(\left(L+F_{1}\right)\left(L+F_{2}\right)^{-1}\right)=1 \text {. }
$$

To show that the Pfaffian is also 1 we may argue as above since the collection of subspaces transverse to the fixed subspace $R(L)$ is path connected (they are all graphs over some fixed transverse subspace). We have finished the proof that the identification of fibers does not depend on the choice of $\operatorname{Cok}(L)$. The proof that this identification does not depend on the choice of $\operatorname{Cok}(D)$ is precisely analogous to the proof just given for $\operatorname{Cok}(L)$ and so we omit this. QED

One consequence of this result is that it is possible to rigorously identify the correlations functions of the Ising model as infinite Pfaffians. To do this it is useful to reinterpret the formulas for the correlations derived in [PT] in terms of a trivialization of the spin bundle over the transfer matrices. It is proved in [PT] that below the critical temperature the $p$-point spin correlations are given by the vacuum expectation of a lift of $T_{\Gamma}(n, N)$ into the spin group. This lift is, of course, a trivialization of the spin bundle, and one may think of the vacuum expectation as the value of the canonical section relative to this trivialization. It would be interesting to characterize the appropriate trivialization directly in the Pfaffian formalism without reference to the transfer matrix formalism. This is connected with $Z / 2 Z$ gauge invariance in a way that we hope to explain in another place.

\section{References}

[AS] Atiyah, M., Singer, I.: Index theory for skew-adjoint Fredholm operators. Publ. Math. IHES, 37, 305-326 (1969)

[B] Baxter, R.: Phil. Trans. Roy. Soc. 289A, 315 (1978)

[CO] Carey, A., O'Brien, D.: Automorphisms of the infinite dimensional Clifford algebra and the Atiyah-Singer mod 2 index. Topology 22, 937-948 (1983)

[CP] Carey, A., Palmer, J.: Infinite complex spin groups. J. Funct. Anal. (to appear)

[H] Hurst, A.: The relation between the Onsager and the Pfaffian method for solving the Ising problem. I. The rectangular lattice. J. Math. Phys. 6, 1 (1965)

[JLW] Jaffe, A., Lesniewski, A., Weitsman, J.: Pfaffians on Hilbert space, Harvard preprint HUTMP B207 Oct. 27, 1987

[K] Kaufman, B.: Crystal statistics II. Partition function evaluated by spinor analysis. Phys. Rev. 76, 1232-1243 (1949)

[MW] McCoy, B. M., Wu, T. T.: The two dimensional Ising Model. Cambridge, MA: Harvard University Press 1973

[O] Onsager, L.: Crystal statistics I. A two dimensional model with an order disorder transition. Phys. Rev. 65, 117-149 (1944)

[P] Palmer, J.: Monodromy fields on $Z^{2}$. Commun. Math. Phys. 102, 175-206 (1985) 
[PT] Palmer, J., Tracy, C.: Two dimensional Ising correlations: Convergence of the scaling limit, Adv. Appl. Math. 2, 329-388 (1981); Two dimensional Ising correlations: The SMJ analysis, Adv. Appl. Math. 4, 46-102 (1983)

[PA] Perk, J., Au-Yang, H.: Solution of the Hirota discrete time Todas lattice equation and the critical correlations in the $Z$-invariant Ising model, Stony Brook preprint

[PS] Pressley, A., Segal, G.: Loop groups. Oxford: Clarendon Press 1986

[Q] Quillen, D.: Determinants of Cauchy-Riemann operators over a Riemann surface. Funct. Anal. Appl. 19, 31-34 (1985)

[S] Simon, B.: Notes on infinite determinants of Hilbert space operators. Adv. Math. 24, 244-273 (1977)

[SW] Segal, G., Wilson, G.: Loop groups and equations of KdV type. Pub. Math. IHES, 61, 5-65 (1985)

[SMJ] Sato, M., Miwa, T., Jimbo, M.: Holonomic quantum fields V. Publ. RIMS, Kyoto Univ. 16, $531-584(1980)$

[W] Witten, E.: Quantum field theory, Grassmannians, and algebraic curves. Commun. Math. Phys. $113,529-600(1988)$

Communicated by K. Gawedzki

Received May 13, 1988; in revised form August 16, 1988 